\title{
White matter lateralization and interhemispheric coherence to auditory modulations in normal reading and dyslexic adults ${ }^{1}$
}

Maaike Vandermosten ${ }^{*, a-c}$, Hanne Poelmans ${ }^{*, a, b}$, Stefan Sunaert ${ }^{c}$, Pol Ghesquière $^{b}$, Jan Wouters ${ }^{a}$

${ }^{*}$ Both authors equally contributed to this study and should be regarded as joint first authors.

${ }^{a}$ ExpORL, Department of Neurosciences, KU Leuven, Herestraat 49, 3000 Leuven, Belgium

${ }^{b}$ Parenting and Special Education Research Unit, KU Leuven, Leopold Vanderkelenstraat 32 - PO Box 3765, 3000 Leuven, Belgium

${ }^{c}$ Radiology Section, KU Leuven, Herestraat 49, 3000 Leuven, Belgium

\section{Corresponding authors:}

Maaike Vandermosten, Leopold Vanderkelenstraat 32 - PO Box 3765, 3000 Leuven, Belgium. Tel.: +32 163261 73, Fax: +32 163259 33, Maaike.Vandermosten@ppw.kuleuven.be Hanne Poelmans, O\&N2, Herestraat 49 - PO Box 721, 3000 Leuven, Belgium. Tel.: +32 163304 95, Fax: +32 163304 86, Hanne.Poelmans@med.kuleuven.be

Keywords: dyslexia, DTI, white matter, ASSR, interhemispheric coherence

Abbreviations: AM: amplitude modulation, ASSR: auditory steady-state responses, DTI: diffusion tensor imaging, FA: fractional anisotropy, CCsplenium: splenium of the corpus callosum, STGp: posterior region of the superior temporal gyrus

\footnotetext{
${ }^{1}$ This article has been published as: Vandermosten, M., Poelmans, H., Sunaert, S., Ghesquière, P., Wouters, J. (2013). White matter lateralization and interhemispheric coherence to auditory modulations in normal reading and dyslexic adults. Neuropsychologia, 51 (11), 2087-2099. doi: 10.1016/j.neuropsychologia.2013.07.008.
} 


\begin{abstract}
Neural activation of slow acoustic variations that are important for syllable identification is more lateralized to the right hemisphere than activation of fast acoustic changes that are important for phoneme identification. It has been suggested that this complementary function at different hemispheres is rooted in a different degree of white matter myelination in the left versus right hemisphere.

The present study will investigate this structure-function relationship with Diffusion Tensor Imaging (DTI) and Auditory Steady-State Responses (ASSR) respectively. With DTI we examined white matter lateralization in the cortical auditory and language regions (i.e. posterior region of the superior temporal gyrus and the arcuate fasciculus) and white matter integrity in the splenium of the corpus callosum. With ASSR we examined interhemispheric coherence to slow, syllabic-rate (i.e. $4 \mathrm{~Hz}$ ) and fast, phonemic-rate (i.e. $20 \mathrm{~Hz}$ ) modulations. These structural and functional techniques were applied in a group of normal reading adults and a group of dyslexic adults for whom previously reduced functional interhemispheric connectivity at $20 \mathrm{~Hz}$ has been reported (Poelmans et al., 2012). This sample was chosen since it is hypothesized that in dyslexic readers insufficient hemispheric asymmetry in myelination might relate to their auditory and phonological problems.

Results demonstrate reduced white matter lateralization in the posterior superior temporal gyrus and the arcuate fasciculus in the dyslexic readers. Additionally, white matter lateralization in the posterior superior temporal gyrus and white matter integrity in the splenium of the corpus callosum related to interhemispheric coherence to phonemic-rate modulations (i.e. $20 \mathrm{~Hz}$ ). Interestingly, this correlation pattern was opposite in normal versus dyslexic readers. These results might imply that less pronounced left white matter dominance in dyslexic adults might relate to their problems to process phonemic-rate acoustic information and to integrate them into the phonological system.
\end{abstract}




\section{INTRODUCTION}

\subsection{Reading \& dyslexia}

Spoken language comprehension depends on the recognition and interpretation of meaningful units, such as words, phrases or sentences. These high-level perceptual constructs are assembled from pre-lexical, acoustic-phonetic cues that are integrated and categorized along multiple timescales. In time windows of $\pm 20-80 \mathrm{~ms}(12-50 \mathrm{~Hz})$ segmental information about for example phoneme identity is clustered, whereas in longer time windows of \pm 150 -300 ms $(3-7 \mathrm{~Hz})$, important suprasegmental information about for example syllables is processed (Obrig, Rossi, Telkemeyer, \& Wartenburger, 2010; Pöppel, Idsardi, \& van Wassenhove, 2008). It is assumed that precise processing of acoustic cues signalling these syllabic- and/or phonemic-rate time windows in speech is crucial for the development of well-specified phonological representations (Nittrouer, 2006). During development, the explicit awareness of these phonological representations changes in grain size. Preschool children manipulate speech mainly at the syllable level, and it is only during reading acquisition, when children learn that every speech sound (phoneme) corresponds to a written symbol (grapheme), that explicit phoneme level representations develop (Anthony, Lonigan, Driscoll, Phillips, \& Burgess, 2003; Ziegler \& Goswami, 2005).

Difficulties in processing and representing phonological information is considered the core deficit in developmental dyslexia (e.g., Snowling, 2000; but see Ramus \& Szenkovits, 2008), a hereditary neurological disorder characterized by severe and persistent reading and/or spelling impairments despite normal intelligence and adequate educational opportunities (Vellutino, Fletcher, Snowling, \& Scanlon, 2004). Yet, it remains unspecified whether these phonological problems in dyslexia only implicate the phoneme- or also the syllable level. According to the traditional view of the phonological deficit theory the locus of the deficit is at the phoneme level (de Gelder \& Vroomen, 1991; Goswami \& East, 2000; Snowling, 2000; Swan \& Goswami, 1997), but some recent theories favour a deficit at the syllable level (Goswami, 2002; Lambrecht Smith, Roberts, Locke, \& Tozer, 2010; Maionchi-Pino, Magnan, \& Ecalle, 2010). In addition, given that several studies have shown that auditory processing skills support a predictive influence on phonological and eventually on reading development (Boets et al., 2011; Corriveau, Goswami, \& Thomson, 2010; Leppänen et al., 2010), it has been suggested that a fundamental deficit in auditory processing indirectly underlies the reading and spelling problems in individuals with developmental dyslexia (e.g., Tallal, 1980; for a review, Farmer \& Klein, 1995). Similarly to the discussion on the locus of the phonological deficit, auditory processing problems were originally thought to involve difficulties in processing brief, rapidly successive and fast dynamic acoustic cues characteristic for phonemes, but some recent theories place the locus of an auditory deficit in the slow changing dynamic cues 
characteristic for syllables (Goswami, 2011). To date, the psychophysical studies are inconsistent as to whether individuals with dyslexia only demonstrate a decreased sensitivity to temporal cues within the syllable range or whether this deficit manifests over a wider range of temporal information (adults: McAnally \& Stein, 1997; Menell, McAnally, \& Stein, 1999; Witton et al., 1998; Witton, Stein, Stoodley, Rosner, \& Talcott, 2002; Stuart, McAnally, Mckay, Johnston, \& Castles, 2006; children: Lorenzi, Dumont, \& Fullgrabe, 2000; Rocheron, Lorenzi, Fullgrabe, \& Dumont, 2002). Defining the neurobiological component of how phonemic-rate and syllabic rate acoustic cues are typically integrated into more abstract phonological units and how this deviates in dyslexic readers can assist in finding the locus of the deficit in dyslexia.

\subsection{Auditory and phonological processing in normal readers}

According to the hierarchical model of speech processing (Hickok \& Pöppel, 2007), early cortical structures such as heschl's gyrus and the supratemporal plane are sensitive to unstructured and simple time-structured acoustic signals respectively, whereas higher level structures such as the superior temporal gyrus are best responsive to complex sublexical spectrotemporal signals and are thought to be involved in the construction of sound-based representations of speech (Hickok \& Pöppel, 2004). Next, at the level of the anterior and posterior superior temporal sulcus an abstract representation of speech units, insensitive to acoustic variances, is obtained (Okada et al., 2010; Peelle, Eason, Schmitter, Schwarzbauer, \& Davis, 2010). From the superior temporal cortex, speech signals are processed in two parallel streams. In a ventral stream, sound-based representations of speech are mapped onto conceptual representations (Rauschecker \& Scott, 2009; Pöppel et al., 2008). This stream engages areas in the left anterior superior temporal sulcus and middle temporal gyrus. It is evidenced that the ventral anatomical counterpart of this sound-to-meaning stream is the extreme capsule fiber system or inferior fronto-occipital fasciculus (Frey, Campbell, Pike, \& Petrides, 2008; Wong, Chandrasekaran, Garibaldi, \& Wong, 2011). In addition, starting from the posterior temporal cortex, a dorsal stream projects towards inferior parietal and frontal (Broca's and premotor) regions and is functionally responsible for mapping phonemic representations onto motor representations (Pöppel et al., 2008) and for sustaining the phonological aspects of speech perception (Chevillet, Jiang, Rauschecker, \& Riesenhuber, 2013; Saur et al., 2010). Saur et al. (2010) demonstrated that the functional connection between the frontal and temporoparietal components is mediated via the arcuate fasciculus (often used interchangeably with the superior longitudinal fasciculus (Brauer, Anwander, \& Friederici, 2011) or as one of its subdivision (Makris et al., 2005)). Thus, phonological processing during speech perception depends both on acoustically-based processes in the posterior temporal areas but also on a dorsal interaction with frontal regions. 
Of special interest with regard to how phonemic- and syllabic-rate acoustic cues are processed is the large body of evidence that the activation of these areas is mainly lateralized to the left hemisphere during the phonetic analysis of speech (for a review see Tervaniemi \& Hugdahl, 2003). According to the domain-driven hypothesis, linguistic aspects of speech drive this lateralization (Dehaene-Lambertz et al., 2005; Dehaene-Lambertz et al., 2010; Rosen, Wise, Chadha, Conway, \& Scott, 2011) whereas the signal-driven hypothesis assumes that spectrotemporal aspects determine the left-right bias. According to the latter hypothesis, spectral acoustic cues and slow temporal modulations are preferentially processed in the right auditory cortex, whereas temporal acoustic analyses, such as the analysis of duration as well as faster modulations, are rather left lateralized (for a review see Pöppel et al., 2008; Zatorre \& Gandour, 2008). According to Pöppel and colleagues (2008), this functional specialization is situated in the non-primary auditory cortex and rooted in the length of integration windows, being between $20-50 \mathrm{~ms}$ (i.e. $12-50 \mathrm{~Hz}$ ) in the left and between $150-300 \mathrm{~ms}$ (i.e. $3-7 \mathrm{~Hz}$ ) in the right hemisphere. Hence, the signal-driven hypothesis explains a left lateralization for phoneme identification (e.g. Jäncke, Wüstenberg, Schulze, \& Heinze, 2002; Dehaene-Lambertz \& Gliga, 2004; Zatorre, Meyer, Gjedde, \& Evans, 1996) and a right lateralization for prosody and syllable identification (Meyer, Alter, Friederici, Lohmann, \& von Cramon, 2002; Zatorre, Evans, Meyer, \& Gjedde, 1992; Zatorre, 1988) based on the length of the integration windows of important acoustic cues instead of their linguistic content (like domain-driven models). Growing experimental evidence supports the claim that not linguistic but rather acoustictemporal characteristics drive hemispheric asymmetry (Boemio, Fromm, Braun, \& Pöppel, 2005; Husain et al., 2006; Joanisse \& Gati, 2003; Zaehle, Wustenberg, Meyer, \& Jancke, 2004). Nevertheless, there remain points of discrepancies across studies such as the observation that phonemes and fast modulations sometimes engage a bilateral activation pattern instead of a clear left dominance (fast modulated non-speech: Belin et al., 1998; Boemio et al., 2005; Pöppel et al., 2008; Telkemeyer et al., 2009; consonant-vowel-combination: Benson, Richardson, Whalen, \& Lai, 2006; Binder et al., 2000; Joanisse et al., 2003). It seems that experience and familiarity with phonetic categories interact with these hemispheric patterns of specialization.

In addition to their functional specialization, the left and right primary auditory and superior temporal cortices also differ in volume (e.g. review Shapleske, Rossell, Woodruff, \& David, 1999), an asymmetry which is mainly present in white rather than in grey matter (Penhune, Zatorre, MacDonald, \& Evans, 1996; Warrier et al., 2009). This finding fits with the hypothesis of Zatorre and colleagues $(2001 ; 2002)$ that the observed functional hemispheric asymmetry for spectrotemporal information is specifically related to hemispheric asymmetry of white matter myelination, rather than to volume asymmetry per se. A more myelinated left auditory cortex would lead to faster neural processing and this would underlie the left specialization for analyzing fast temporal information. 
Direct evidence for white matter lateralization is provided by a post-mortem analysis of the posterior superior temporal lobe, which confirmed the greater white matter volume in the left than in the right hemisphere and showed that this difference was due to greater myelin sheath thickness in the left hemisphere (Anderson, Southern, \& Powers, 1999). A non-invasive MRI-technique that is relatively sensitive to white matter microstructure in vivo is diffusion tensor imaging (DTI). DTI provides parameters, such as fractional anisotropy (FA) which indirectly measures the degree of myelination and/or axon density (Beaulieu, 2002), and is adept to virtually reconstruct white matter pathways in vivo, via tractography (Basser, Pajevic, Pierpaoli, Duda, \& Aldroubi, 2000). To date, evidence is missing on white matter lateralization of the auditory regions, measured by DTI. It has, however, been repeatedly demonstrated that the dorsal white matter tract departing from these regions, the arcuate fasciculus, has a leftward asymmetry in fractional anisotropy (Thiebaut de Schotten et al., 2011; Rodrigo et al., 2007; Büchel et al., 2004; Catani et al., 2007; Barrick, Lawes, Mackay, \& Clark, 2007; Eluvathingal, Hasan, Kramer, Fletcher, \& Ewing-Cobbs, 2007; Hagmann et al., 2006; Matsumoto et al., 2008; Makris et al., 2005; Nucifora, Verma, Melhem, Gur, \& Gur, 2005; Powell et al., 2006; Parker et al., 2005; Vernooij et al., 2007). This suggests that auditory regions, which are important for processing temporal modulations in speech, may also demonstrate a left hemispheric white matter lateralization.

\subsection{Auditory and phonological processing in dyslexic readers}

A large body of evidence allocates the well-known phonological problems in dyslexia to a functional and structural deficit in left temporoparietal areas (reviews by: Eden \& Zeffiro, 1998; Shaywitz, 1998; Shaywitz et al., 2002; meta-analysis: Richlan, Kronbichler, \& Wimmer, 2009) and in the arcuate fasciculus (Vandermosten et al., 2012). Less is known however about the neural correlates of the auditory processing problems that are often observed in dyslexic readers. Several studies assign the auditory problems in dyslexia to deviant hemispheric specialization of the auditory system to process rapidly versus slowly changing acoustic cues. Functional imaging studies have indeed demonstrated a leftward bias for fast compared to slow transitions in normal readers, whereas this asymmetry was not present in their dyslexic peers (Gaab, Gabrieli, Deutsch, Tallal, \& Temple, 2007; Ruff, Cardebat, Marie, \& Démonet, 2002). Recently, it has been proposed that this asymmetry is rooted in a similar hemispheric asymmetry of spontaneous neural oscillatory activity at frequencies near the syllabic (i.e. theta band activity, 3-6 Hz) and the phonemic (i.e. gamma band activity, $28-40 \mathrm{~Hz}$ ) rates and that a disturbance in the spontaneous oscillations in these frequency bands may lead to auditory processing problems observed in dyslexia (Goswami, 2011). Indeed, several EEG and MEG studies provided evidence that in dyslexic readers fast acoustic changes at the 
phonemic-rate were less lateralized to the left (Lehongre, Ramus, Villiermet, Schwartz, \& Giraud, 2011) and slow syllabic-rate modulations are less lateralized to the right hemisphere (Abrams, Nicol, Zecker, \& Kraus, 2008; 2009; Hämäläinen, Rupp, Soltész, Szücs, \& Goswami, 2012). In addition to lateralization differences, we have shown in a previous study that lower left hemispheric responses to phonemic-rate modulations were accompanied by lower intra- and interhemispheric coherence in dyslexic readers, suggesting that phonemic-rate information is less well integrated in the dorsal phonological system in dyslexic adults (Poelmans et al., 2012). These studies indicate that a disturbance in hemispheric lateralization of syllabic- and/or phonemic-rate modulations is present in dyslexia and raises the possibility that the connection of the auditory system with the phonological system is not optimal in dyslexia.

Concerning structural asymmetry in normal versus dyslexic readers, there is mixed evidence for a decreased left asymmetry in the adjacent planum temporale (for reviews see Beaton, 1997; Eckert \& Leonard, 2000; Morgan \& Hynd, 1998). Though volume measures are a valuable index for asymmetry, we expect especially a reduced asymmetry in microstructural white matter properties, such as myelination, to be at the basis of the temporal processing problems in dyslexic readers. In general, DTI-studies provide only indirect evidence for this hypothesis by showing group differences in the white matter microstructure in the left and often not in the right hemisphere (for a review see Vandermosten, Boets, Sunaert, Wouters, \& Ghesquière, 2012). More direct evidence for reduced lateralization in white matter microstructure, as calculated by a lateralization index for segments of several white matter tracts, was provided by Niogi \& McCandliss (2006). In their study, a significantly lesser degree of left lateralization in the temporoparietal region (i.e. superior corona radiata) in dyslexic relative to normal readers was demonstrated. However, no evidence is available on group differences in laterality concerning the full arcuate fasciculus and its projection point to auditory regions (i.e. posterior part of STG).

\subsection{Hypothesis and aims of the present study}

Based on the above-cited evidence for a complementary function of each hemisphere in processing slow and fast temporal modulations and the suggestion that this is rooted in hemispheric differences of myelination, we hypothesize that dyslexics might have an insufficient leftwards myelination asymmetry that relates to their auditory temporal processing problems. However, in order to verify this hypothesis, evidence is lacking on three crucial points. First, more evidence is required on hemispheric differences in white matter, and especially in myelination, of regions involved in auditory processing. As mentioned in 1.2, asymmetries in the volumes of the auditory cortex and its surrounding areas have been observed. However, DTI-studies which investigate 
hemispheric differences in microstructural white matter properties (including myelination) only exist for the arcuate fasciculus and not for its projection point to auditory regions (STGp). Moreover, as mentioned in 1.3 , it is not known whether dyslexic readers have the same degree of white matter asymmetry as normal readers in the arcuate fasciculus and STGp. Second, in normal readers evidence is lacking on the direct relationship between functional neural specialization for slow and fast temporal modulations on the one hand and structural measures, such as the lateralization of myelination in regions responsible for processing this information, on the other hand. Only one study investigated spectrotemporal specialization of each hemisphere in relation to the anatomy of the auditory regions (Warrier et al., 2009). Yet, this study focused on volume parameters (instead of myelination) and on primary auditory regions (instead of secondary auditory regions, where hemispheric specialization for spectrotemporal acoustic information is primarily expected (Pöppel et al., 2008; Zaehle, Geiser, Alter, Jancke, \& Meyer, 2008)). Third, no evidence is available on this function-structure relationship in dyslexic readers. Given that dyslexic readers are impaired in processing rapidly changing signals and that they tend to demonstrate decreased volumetric and functional lateralization, it is interesting to investigate whether lateralization of white matter integrity in the auditory regions and dorsal tract is also reduced in dyslexic readers, and whether the relation between these structural lateralization indices and functional specialization of each hemisphere differs from normal readers.

Therefore, the first aim of the present study was to investigate microstructural white matter lateralization in auditory and language regions in normal reading and dyslexic adults by means of DTI. DTI is a structural MRI technique that provides a quantitative index of the degree of anisotropy, i.e. FA, which is to some extent determined by microstructural white matter properties. Yet, it is important to note that, due to the large voxel size of DTI, the extracted FA-index does not relate directly to myelination properties but is simultaneously sensitive to a variety of microstructural effects of which especially axon properties play a crucial role (Alexander, Pierpaoli, Basser, \& Gee, 2001). We are particularly interested in FA of the posterior region of STG (STGp), since this region is involved in acoustic spectrotemporal analysis as well as in the arcuate fasciculus, since the integration of acoustic information into phonological representations takes place here. Additionally, FA of the callosal projections via the splenium may be important, since these may underlie hemispheric asymmetry in STGp (Westerhausen, Grüner, Specht, \& Hugdahl, 2009). Second, the central aim is to investigate whether FA-lateralization in the dorsal fiber tract and its posterior projection point (arcuate fasciculus and STGp) and FA in the splenium of the corpus callosum is related to neural processing of slow, syllabic-rate and faster, phonemic-rate modulations. In a previous study, which involved an extended sample of the present study, we have shown that adults with dyslexia demonstrate reduced left hemispheric phase-locking to phonemic-rate modulations 
and that this decrease was accompanied by lower intra- and interhemispheric coherence in dyslexic readers (Poelmans et al., 2012). Given that the integration of auditory information into the dorsal phonological system depends on a large network of intra- and interhemispheric connections, it is interesting to examine whether interhemispheric coherence to syllabic- and phonemic-rate modulations is related to FA-lateralization of the dorsal fiber tract and its posterior projection point and with FA in the splenium of the corpus callosum. By also including dyslexic readers, it will be possible to examine whether this correlational pattern was different for both groups.

\section{METHODS}

\subsection{Participants}

Twenty dyslexic and 20 normal reading adults (age range 19-32 years), recruited from the student population of the University of Leuven, participated. All were native Dutch speakers, without a history of brain damage, language problems, psychiatric symptoms, visual problems or hearing loss. All participants were predominantly right-handed as assessed by the Edinburgh Handedness Inventory (Oldfield, 1971), had adequate nonverbal intelligence (standard score $>90$ on the Matrices subtest of WAIS-III (Wechsler, 1992)), and adequate audiometric pure-tone hearing thresholds $(<25$ $\mathrm{dB} H \mathrm{HL}$ on all octave audiometric frequencies). The 40 participants of the present study were identical to the sample described in Vandermosten et al. (2012) and partly overlap with the sample described in Poelmans et al. (2012). All individuals with dyslexia had a formal diagnosis of developmental dyslexia and scored below the $3^{\text {rd }}$ percentile of a university norm group (Depessemier \& Andries, 2009) on both standardized reading tests. The 20 normal reading individuals reported no history of reading difficulties and scored above the $20^{\text {th }}$ percentile of the university norm group on both reading tests. Both groups were matched for gender, age and nonverbal intelligence. As defined, performance on word and pseudoword reading and spelling was significantly poorer in the dyslexic compared to the normal reading group. Table 1 displays descriptive statistics for the two groups. The study was approved by the local Ethical Board and informed consent was obtained from all participants according to the Declaration of Helsinki. 
Table 1 Participant characteristics

\begin{tabular}{lccc}
\hline & $\begin{array}{c}\text { Dyslexic readers } \\
(N=20)\end{array}$ & $\begin{array}{c}\text { Normal readers } \\
(N=20)\end{array}$ & Test statistics \\
\hline & $\mathrm{M}(\mathrm{SD})$ & $\mathrm{M}(\mathrm{SD})$ & \\
\hline Subject characteristics & & & \\
Gender (male/female) & $7 / 13$ & $8 / 12$ & $\chi^{2}(1)=0.11, p=0.744$ \\
Age (years) & $22.1(3.1)$ & $21.4(3.0)$ & $t(38)=-0.66, p=0.514$ \\
Non-verbal IQ & $108(10)$ & $106(10)$ & $t(38)=-0.54, p=0.592$ \\
Defining literacy measures & & & \\
Word reading & & $99.8(11.4)$ & $t(38)=13.10, p<0.0001$ \\
Pseudoword reading & & $107.9(9.8)$ & $t(38)=18.75, p<0.0001$ \\
Spelling $^{\mathrm{a}}$ & $66.1(1.9)$ & $105.8(9.6)$ & $t(38)=14.04, p<0.0001$ \\
\hline
\end{tabular}

${ }^{\mathrm{a}}=$ Standardized scores with population average $\mathrm{M}=100$ and $\mathrm{SD}=15$

\subsection{Structural measures: DTI}

\subsubsection{Data acquisition}

All participants underwent MRI examination on a 3T system (Philips, Best, The Netherlands). The DTI data were acquired using a single spin shot EPI with SENSE acquisition. DTI images covering the entire brain and the brainstem were acquired with the following parameters: matrix size $=112 \mathrm{x}$ 109; field of view $(F O V)=220 \times 220 \mathrm{~mm}^{2}$; repetition time $(T R)=11043 \mathrm{~ms}$, echo time $(T E)=55 \mathrm{~ms}, 68$ contiguous sagittal slices (slice thickness $=2.2 \mathrm{~mm}$; voxel size $=1.96 \times 1.96 \times 2.2 \mathrm{~mm}^{3}$ ), acquisition time $=10 \mathrm{~min} 34 \mathrm{~s}$. A pair of diffusion gradients was applied along 45 non-collinear directions with a $\mathrm{b}$-value of $800 \mathrm{~s} / \mathrm{mm}^{2}$. Additionally, one set of images with no diffusion weighting $\left(\mathrm{b}=0 \mathrm{~s} / \mathrm{mm}^{2}\right)$ was acquired. Two identical DTI datasets were consecutively acquired for each subject to increase the signal-to-noise ratio, bringing the total acquisition time to $21 \mathrm{~min}$ and $8 \mathrm{~s}$.

\subsubsection{Data processing}

DTI images were first visually checked for possible artefacts. DTI post-processing involved correcting for eddy-current and motion-induced artefacts using CATNAP with the required reorientation of the b-matrix (Landman et al., 2007; Leemans \& Jones, 2009). Fractional anisotropy (FA) was extracted from 2 left and right brain regions: the posterior part of the Superior Temporal gyrus (STGP) and the arcuate fasciculus (Figure 1). In addition, we delineated the fibers passing through the splenium of the corpus callosum (CCsplenium) (Figure 1). This posterior part of the CC was chosen as it is demonstrated that it connects the bilateral STGp (Westerhausen et al., 2009). Concerning fibertracking for the arcuate fasciculus and CCsplenium, a whole brain tractography was 
first calculated for each concatenated DTI dataset using a seed point of [2 2 2], fractional anisotropy (FA) threshold of 0.2 to seed and end tracking, angle threshold of $40^{\circ}$, and fiber length range of 50 $500 \mathrm{~mm}$. Fibertracking of arcuate fasciculus and CCsplenium was done in native space and an average FA was obtained per tract. To reconstruct the arcuate fasciculus, we defined the regions of interest in line with the protocol of Wakana et al. (2007), there referred to as 'SLF', and for CCsplenium, the splenium was delineated on the midsagittal slice in line with the protocol of Witelson (1989). Concerning the bilateral STGp, the individual concatenated datasets were nonrigidly normalized to MNI (Montreal Neurological Institute) space using the software ExploreDTI (Leemans et al., 2009). In addition, FA-data were smoothed with a $6 \mathrm{~mm}$ Gaussian kernel. Each participant's DTI data were masked based on their individual FA map at a threshold of 0.15 in order to restrict the analyses to white matter. Subsequently, the region of interest of STGp was defined based on the Harvard-Oxford atlas in MNI space at 25\% probability threshold. The region of interest of STGp was placed on the smoothed masked normalized FA-maps of each individual, and FA-values were extracted.

Next, given our focus on structural asymmetry in white matter integrity, we calculated a lateralization index (LAT) for the arcuate fasciculus and STGp according to the following formula:

$$
\operatorname{LAT}(F A)=\frac{\operatorname{right}(F A)-\operatorname{left}(F A)}{\operatorname{right}(F A)+\operatorname{left}(F A)} \times 100
$$

A negative lateralization score indicates the FA is relatively left lateralized across two regions of interest while a positive lateralization score reflects relative right FA-lateralization. Values close to zero indicate no lateralization.

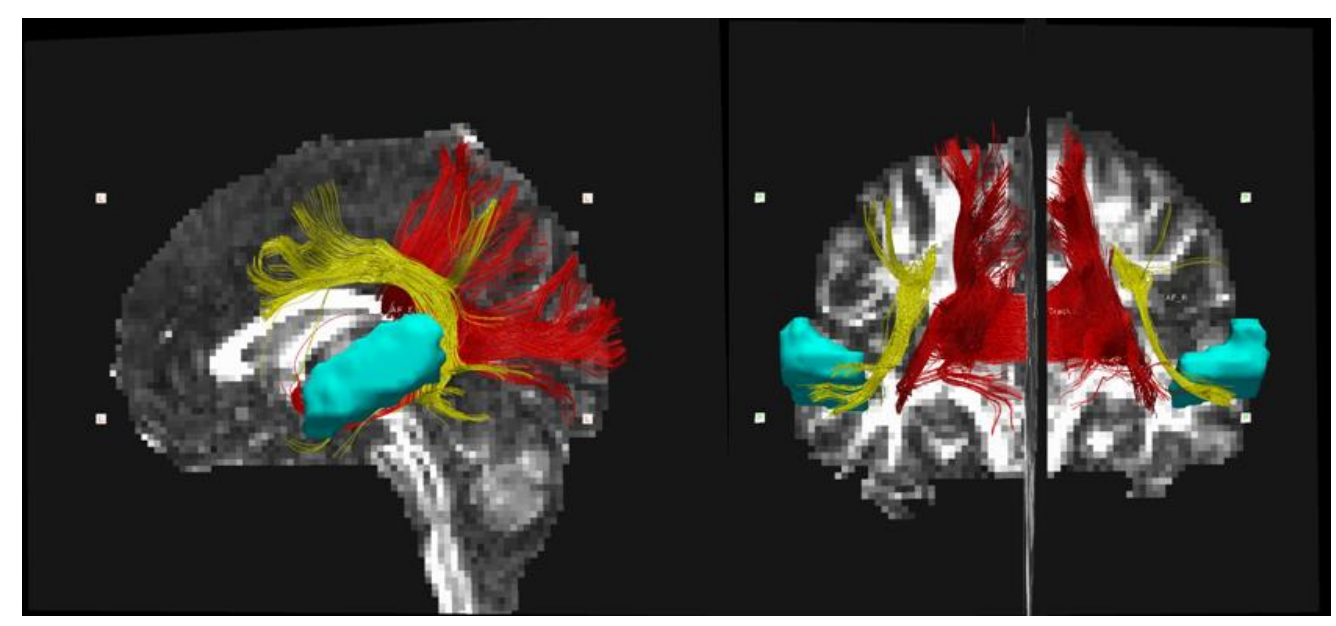

Figure 1 Left arcuate fasciculus (depicted in yellow), STGp (depicted in light blue), and CCsplenium (depicted in red). Left panel: sagittal view from the left. Right panel: coronal view from posterior. 


\subsection{Electrophysiologic measures: ASSR}

Neural sensitivity to syllabic- and phonemic-rate modulations was investigated by means of auditory steady-state responses (ASSRs) in the EEG. The ASSR is a neural oscillatory activity that is evoked by a rhythmic auditory stimulus (Picton, John, Dimitrijevic, \& Purcell, 2003; 2011). ASSRs measure how well the auditory system phase-locks to the stimulus rhythm (by means of response strengths) and how well neural oscillations in both hemispheres cohere in their response to syllabicand phonemic-rate modulations (by means of phase coherence). ASSRs were recorded in the same sample of participants as the DTI recording. This participant sample is a subgroup of the sample reported in Poelmans et al. (2012).

\subsubsection{Stimuli}

ASSRs were recorded to continuous amplitude modulated speech-weighted noise stimuli. The noise carriers were derived from the speech-weighted masking noise of the "Leuven Intelligibility Sentence Test" (LIST: van Wieringen \& Wouters, 2008). This noise represents the long-term average speech spectrum of 730 sentences of a female speaker. Noises were $100 \%$ amplitude modulated at $3.91 \mathrm{~Hz}$ and $19.53 \mathrm{~Hz}$. For simplicity, these modulation frequencies will be further referred to as the rounded frequencies: $4 \mathrm{~Hz}$ and $20 \mathrm{~Hz}$ respectively. These modulation frequencies were selected because of their importance in speech intelligibility (Shannon, Zeng, Kamath, Wygonski, \& Ekelid, 1995), representing the syllabic- and phonemic-rate respectively. Stimuli were generated in Matlab R14. In the experiment, all stimuli were presented monaurally to the right ear with ER-3A insert phones, at $70 \mathrm{~dB}$ SPL.

\subsubsection{Data acquisition}

The continuous EEG was recorded by 10 surface $\mathrm{Ag} / \mathrm{AgCl}$ electrodes on the scalp, placed in accordance with the international 10-20 system for electrode placement (Malmivuo \& Plonsey, 1995). EEG was recorded from two central electrodes, Oz and Fpz, three left hemispheric electrodes, P3, F3 and M1 (left mastoid) and three corresponding right hemispheric electrodes, P4, F4 and M2 (right mastoid). The reference electrode was placed at $\mathrm{Cz}$ and the ground electrode the right clavicle. Inter-electrode impedances were kept below $5 \mathrm{kOhm}$ at $30 \mathrm{~Hz}$. The electrodes were connected to a low-noise Jaeger-Toennies multichannel amplifier. Each EEG channel was amplified with a gain of 50000 and band-pass filtered between 0.2 and $100 \mathrm{~Hz}(6 \mathrm{~dB} /$ octave) for $4 \mathrm{~Hz}$ and between 2 and $100 \mathrm{~Hz}$ ( $6 \mathrm{~dB}$ /octave) for the $20 \mathrm{~Hz}$ modulation. Because the ASSR is a frequencyspecific response, these filter settings only served to minimize the number of artefacts in the EEG. The amplified EEG signals were recorded and stimuli were presented by a RME Hammerfall DSP Multiface multichannel soundcard in combination with the experimental Setup ORL for Multichannel ASSR (SOMA) (Van Dun et al., 2008) at a sampling rate of $32 \mathrm{kHz}$ and downsampled to $1 \mathrm{kHz}$. The 
continuous EEG was recorded in epochs. Each epoch consisted of 1024 samples (corresponding to 1.024 seconds). Sixteen successive epochs were clustered into a sweep. Online artifact rejection was set to $100 \mu \mathrm{V}$, to exclude muscle artefacts. For each stimulus, 18 artifact-free sweeps were recorded. The EEG was recorded in a double-walled and soundproof booth with a Faraday cage. Participants were asked to lie down on a bed and to watch a soundless movie to stay alert. Two ASSR recordings were carried out for each stimulus within the same session.

\subsubsection{Data processing}

Data analyses were performed by means of Matlab R14. Ten percent of the recorded epochs, i.e., those with the largest noise values, in each recording were rejected to exclude artefacts. Sweeps were reconstructed by clustering 16 consecutive noise-free epochs. For each recording, the remaining 16 sweeps of EEG were weighted-averaged in the time domain (John, Dimitrijevic, \& Picton, 2001) and transformed into the frequency domain using a Fast Fourier Transform (FFT). Because the test and retest did not differ significantly, both recordings were linked together and FFT statistics were carried out over the linked data, i.e., after 32 sweeps. Data-analyses was performed on response-SNRs of parietal (P3, P4) and mastoid electrodes (M1, M2) (for a detailed description of the data analysis see: Poelmans et al., 2012). For these electrode pairs interhemispheric phase coherence was calculated, according to the following formula:

$$
C_{12}(f)=\frac{\left|W_{12}(f)\right|^{2}}{\left(W_{11}(f) \times W_{22}(f)\right)}
$$

Phase coherence is based on the degree of coherence between neuronal oscillatory networks in both hemispheres and is a sensitive marker of functional connectivity between distant brain regions. Coherence analyses are based on the idea that brain regions involved in the same process show increased coherence (i.e. neuronal cooperation) in order to integrate processed information into integrated constructs (Weiss \& Mueller, 2003), and present a measure of a consistent relationship between the amplitude-fluctuations of two regions, irrespective of their phase. Phase coherence is calculated as a correlation in the time domain between two signals, recorded simultaneously at different scalp electrodes at a given frequency (or frequency band). The coherence at frequency $f$ between signals 1 and 2 indicated as $C_{12}(f)$ is calculated as the squared magnitude of the cross-spectral density of the signal pair $W_{12}(f)$, normalized by dividing with the product of the power spectra of the two signals, respectively $W_{11}(f)$ and $W_{22}(f)$ (Nunez \& Srinivasan, 2006). Coherence values range between zero and one. Low interhemispheric coherence indicates no coherence between active neural networks in the left and the right hemispheres, whereas high 
coherence indicates a high degree of coherent neuronal activity between both hemispheres. For $4 \mathrm{~Hz}$ and for $20 \mathrm{~Hz}$, interhemispheric phase coherence was calculated separately for parietal and mastoid electrodes and averaged into one measure for interhemispheric coherence to each modulation rate.

Because of the small number of electrodes, it is important to note that these coherence analyses are limited to sensor space. Sensor space analyses can be influenced by the location of the underlying source(s) and by factors such as volume conductance. However, given the large distance between the analyzed electrodes and the assumed underlying sources (e.g. Herdman et al., 2002; Millman, Prendergast, Kitterick, Woods, \& Green, 2010; Lehongre et al., 2011), the effect of source location and volume conductance are thought to be minimal. To additionally decrease the influence of these possible confounding factors, ASSR results are only compared between groups and separately for the $4 \mathrm{~Hz}$ and $20 \mathrm{~Hz}$ modulation rates, and not interpreted in terms of absolute coherence values.

\subsubsection{ASSR-results}

In a previous study (Poelmans et al., 2012), with an overlapping but larger sample as in the present study, we reported that adults with dyslexia demonstrated reduced phase coherence between the left and the right hemisphere for phonemic-rate but not for syllabic-rate responses (Poelmans et al., 2012). That is, normal reading adults demonstrated higher interhemispheric coherence than dyslexic adults for $20 \mathrm{~Hz}$ (mean difference: 0.11 , SE: 0.05, $p=0.040$ ). For $4 \mathrm{~Hz}$, on the other hand, interhemispheric coherence did not differ between groups (mean difference: $-0.02, \mathrm{SE}$ : $0.03, p=0.516)$.

\subsection{Statistical analyses}

First, in order to investigate whether normal and dyslexic readers showed significant FAlateralization in STGp and in arcuate fascisulus, a one sample t-test was applied. Next, to investigate group differences in FA-lateralization of STGp and arcuate fasciculus and in FA of CCsplenium, a general linear model was applied with group as explanatory variable and with two covariates, i.e. IQ and the quality of the DTI data acquisition (i.e. indexed by mean chi square). Finally, in order to assess the link of the DTI-measures with the two ASSR-measures, we added $4 \mathrm{~Hz}$ interhemispheric coherence and $20 \mathrm{~Hz}$ interhemispheric coherence as well as their interaction with group as explanatory variables to the general linear modal that already contained group and the two covariates. To interpret the observed effects, a correlation matrix between the DTI-indices and the ASSR-indices (with IQ and mean chi-square as covariates) are provided as well as the scatterplots of these residuals. After visual inspection of these scatterplots, the correlations in normal readers between $20 \mathrm{~Hz}$ ASSRs and STGp and between $20 \mathrm{~Hz}$ ASSRs and CCsplenium seemed to be driven by outlying values, i.e. standardised residual of $>1.5$ SD on both variables (see green and orange circles 
in Figure 4, upper panel). To investigate whether correlations were confirmed when removing these three outlying values, we reran the correlations (Figure 4, lower panel).

\section{RESULTS}

\subsection{DTI-indices (FA-lateralization of STGp and arcuate fasciculus, and FA in CCsplenium) in dyslexic versus normal readers}

A lateralization index based on the left and right FA-values in STGp and arcuate fasciculus was calculated and compared between dyslexic and normal readers. In addition, FA of CCsplenium was extracted and compared between both groups. Concerning the STGp, normal readers demonstrated a significant left FA-lateralization $(t(19)=-4.53, p<0.001)$, whereas dyslexic readers rather showed a symmetric FA-distribution $(t(19)=-0.26, p=0.801)$. Group comparison confirmed that dyslexic readers had a significantly lower degree of left lateralization than normal readers $(F(1,36)=5.79, p=0.021)$ (Figure 2, left panel). This group difference in STGp lateralization was rooted in a non-significant tendency of a lower FA in the left and a higher FA in the right STGp in dyslexic relative to normal readers $(p>0.17)$. The individual distribution of lateralization indices is displayed in the left panel of Figure 3, and indicates that dyslexic readers have a lower lateralization index than normal readers, and additionally, a lower number of dyslexic individuals have a left lateralization (i.e. a negative lateralization index in 17 of the 20 normal readers versus 12 of the 20 dyslexic readers).

Concerning the arcuate fasciculus, we described in a previous paper (Vandermosten et al., 2012) that dyslexic readers showed a significant lower FA in the left arcuate fasciculus but not in the right compared to normal readers. This could suggest a group difference in the lateralization index, which we calculated in the present study. Results indicated a significant left lateralization for both normal reading $(t(19)=-7.34, p<0.001)$ and dyslexic $(t(19)=-6.51, p<0.001)$ readers, but this left FA-lateralization was again less prominent in dyslexic than in normal readers $(F(1,36)=4.39$, $p=0.043$ ) (Figure 2, right panel). The individual lateralization distribution (Figure 3, right panel) demonstrates that almost every normal and dyslexic reader exhibits a left lateralization (i.e. negative lateralization index), but that dyslexic readers show this to a lesser extent.

For the CCsplenium no group differences in FA were found between normal reading and dyslexic adults $(F(1,36)=0.19, p=0.668)$.

To conclude, while the CCsplenium did not demonstrate a difference between normal reading and dyslexic adults, group differences were found in the lateralization of STGp and arcuate fasciculus. More specifically, STGp and arcuate fasciculus display a left FA-lateralization in normal 
readers, whereas in dyslexic readers a symmetric FA-pattern in STGp and a less pronounced left FAlateralization in arcuate fasciculus is observed.

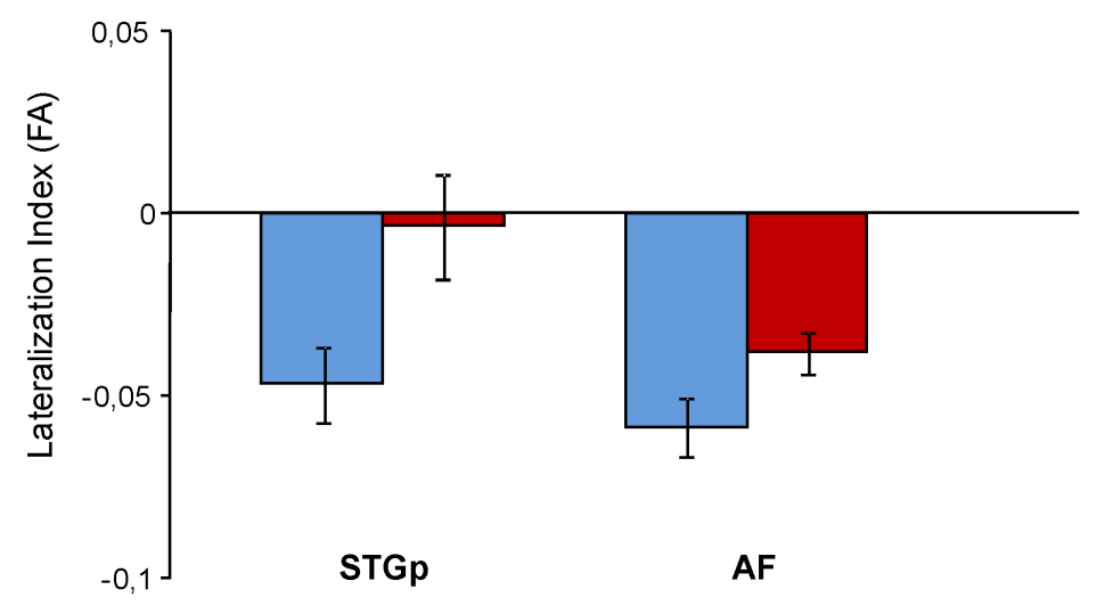

Figure 2 Average FA-lateralization indices of STGp and arcuate fasciculus, which showed a significant difference between normal (blue bars) and dyslexic (red bars) readers. Error bars indicate \pm 1 SE.

STGp
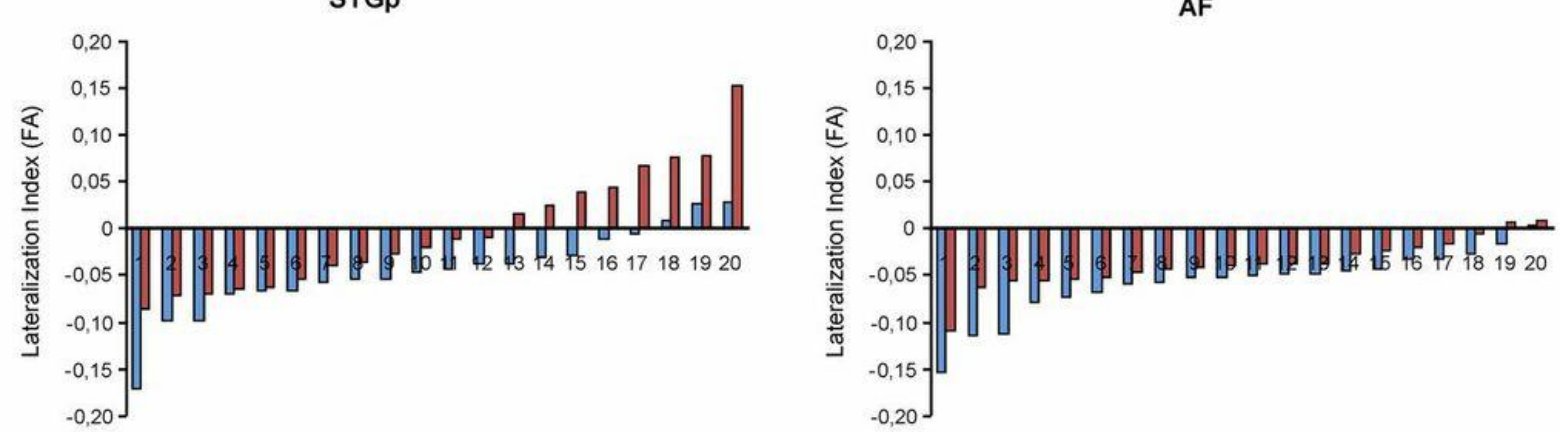

Figure 3 Individual distribution of the lateralization indices of the STGp (blue bars) and arcuate fasciculus (red bars) for normal and dyslexic readers. 


\subsection{Relation between DTI-indices and neural processing of syllabic- and phonemic-rate modulations}

In a second step, we investigated the structure-function relationship for each group. To test this, each DTI-index (i.e. FA of CCsplenium and FA-lateralisation of STGp and arcuate fasciculus) is predicted on the basis of group, the two ASSR-interhemispheric coherence indices (i.e. $4 \mathrm{~Hz}$ and $20 \mathrm{~Hz} \mathrm{AM}$ ) and their interaction with group.

For arcuate fasciculus, no significant main effects of interhemispheric coherence to $4 \mathrm{~Hz}$ $(F(1,39)=0, p=0.953)$ and to $20 \mathrm{~Hz} \operatorname{AM}(F(1,39)=0.03, p=0.874)$, nor an interaction of them with group was found (group x $4 \mathrm{~Hz}: F(1,39)=0, p=0.963$; group $\times 20 \mathrm{~Hz}: F(1,39)=1.08, p=0.305$ ). This is also reflected in no significant correlations of arcuate fasciculus with neural responses to $4 \mathrm{~Hz}$ and $20 \mathrm{~Hz}$ modulations in normal readers and dyslexic readers (see Table 2).

For STGp, no significant main effects of interhemispheric coherence to $4 \mathrm{~Hz}(F(1,39)=0.09$, $p=0.768)$ and to $20 \mathrm{~Hz} \operatorname{AM}(F(1,39)=0.78, p=0.384)$, nor an interaction between interhemispheric coherence to $4 \mathrm{~Hz}$ and group $(F(1,39)=2.51, p=0.123)$ was found. In contrast, a significant interaction was observed between interhemispheric coherence to $20 \mathrm{~Hz}$ and group $(F(1,39)=6.22$, $p=0.018$ ), implying an opposite contribution for each group on the lateralization of STGp. Indeed, a correlational analysis demonstrated a negative correlation coefficient between FA-lateralization in STGp and interhemispheric coherence to $20 \mathrm{~Hz}$ in normal readers $(r=-0.44, p=0.068)$, whereas in dyslexic readers a positive correlation coefficient was found $(r=0.35, p=0.157$ ) (Table 2 and Figure 4 , upper panel). Given that this negative correlation in normal readers seemed to be driven by one outlying value (i.e. orange circle in Figure 4, upper panel), we reran the correlational analyses. Removal of this participant confirmed, and even strengthened, the observed correlation between $20 \mathrm{~Hz}$ ASSRs and STGp in normal readers $(r=-0.57, p=0.016)$ (Figure 4, lower panel). Overall, these analyses suggest that stronger interhemispheric coherence to $20 \mathrm{~Hz}$ AM coheres with left FAlateralization in STGp for normal readers but with right FA-lateralisation in STGp for dyslexic readers (Figure 4).

For CCsplenium, no signficiant contribution was observed of interhemispheric coherence to $4 \mathrm{~Hz}(F(1,39)=1.39, p=0.247)$ and to $20 \mathrm{~Hz} \operatorname{AM}(F(1,39)=0.21, p=0.654)$, nor an interaction between interhemispheric coherence to $4 \mathrm{~Hz}$ and group $(F(1,39)=0.27, p=0.606)$. On the other hand, a significant interaction was observed between interhemispheric coherence to $20 \mathrm{~Hz} A M$ and group $(F(1,39)=5.80, p=0.022)$, implying again an opposite contribution for each group. More specifically, normal readers showed a negative correlation coefficient between FA in CCsplenium and interhemispheric coherence to $20 \mathrm{~Hz}$ AM $(r=-0.41, p=0.094)$ whereas for dyslexic adults a positive correlation coefficient was found ( $r=0.47, p=0.051$ ) (Table 2 and Figure 4 , upper panel). In order to verify that the correlation in normal readers was not driven by 2 outlying values in the normal 
reading group (i.e. green circles in Figure 4), we reran the correlational analyses and this resulted in an even stronger correlations ( $r=-0.78, p=0.004$ ) (Figure 4 , lower panel). In sum, this indicates that in normal readers, stronger interhemispheric coherence to $20 \mathrm{~Hz} A M$ is related to decreased FA in CCsplenium, whereas stronger interhemispheric coherence to $20 \mathrm{~Hz}$ AM related to higher CCsplenium FA in dyslexic readers.

Table 2 Correlations of interhemispheric coherence to $4 \mathrm{~Hz}$ and $20 \mathrm{~Hz}$ with FA-lateralization of STGp and arcuate fasciculus and with FA of the CCsplenium in normal (NR) and dyslexic readers (DR).

\begin{tabular}{|c|c|c|c|c|c|c|c|c|}
\hline & \multicolumn{4}{|c|}{ Coherence $4 \mathrm{~Hz}$ AM } & \multicolumn{4}{|c|}{ Coherence $20 \mathrm{~Hz}$ AM } \\
\hline & \multicolumn{2}{|c|}{$N R$} & \multicolumn{2}{|c|}{$D R$} & \multicolumn{2}{|c|}{$N R$} & \multicolumn{2}{|c|}{$D R$} \\
\hline & $r$ & $p$ & $r$ & $p$ & $r$ & $p$ & $r$ & $p$ \\
\hline Arcuate Fasciculus & -0.15 & 0.546 & -0.04 & 0.858 & -0.25 & 0.312 & 0.19 & 0.450 \\
\hline STGp & 0.07 & 0.776 & -0.28 & 0.267 & -0.43 & 0.068 & 0.35 & 0.157 \\
\hline CCsplenium & -0.11 & 0.658 & 0.19 & 0.451 & -0.41 & 0.094 & 0.47 & 0.051 \\
\hline
\end{tabular}




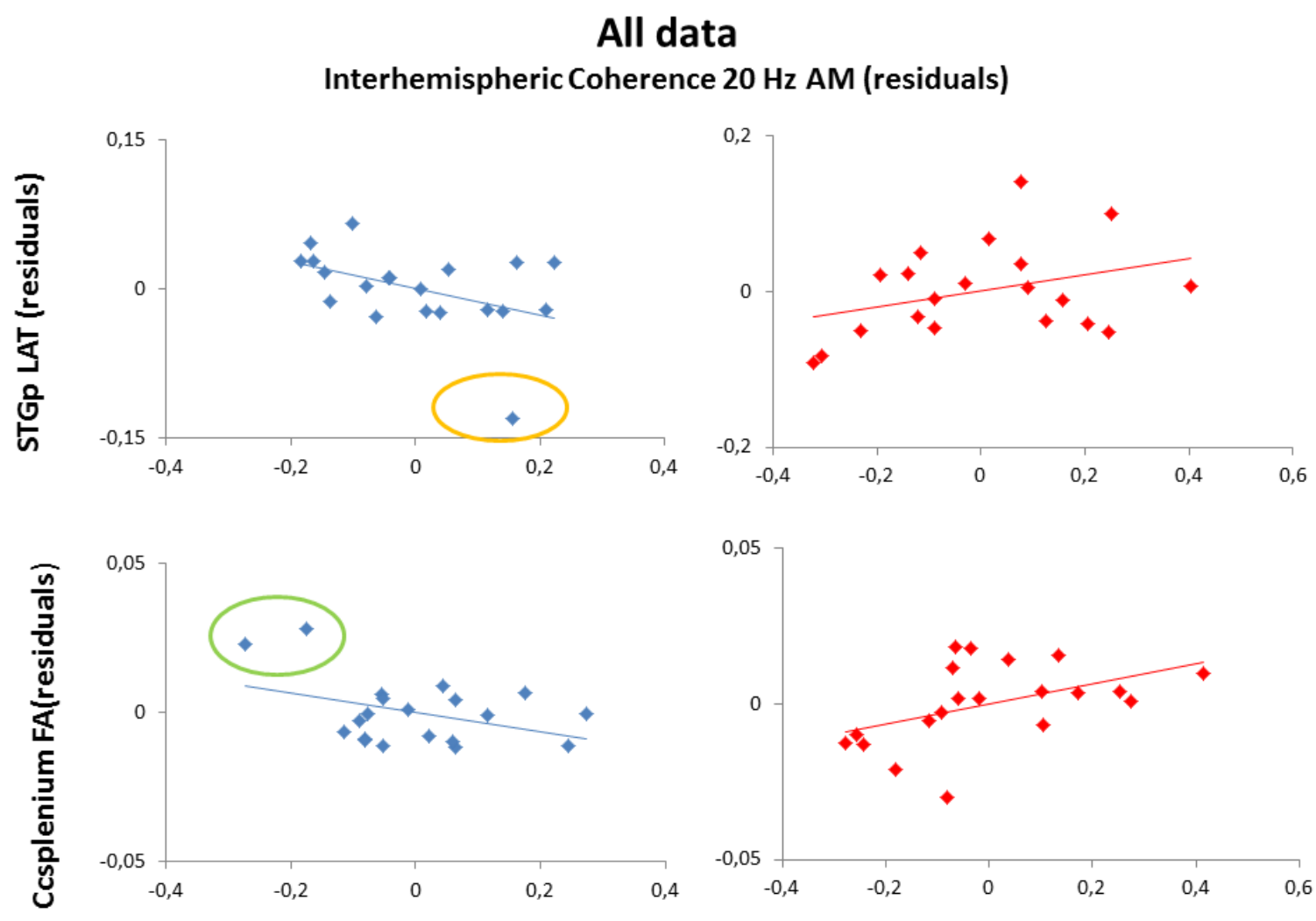

Without outliers:

Interhemispheric Coherence $20 \mathrm{~Hz}$ AM (residuals)
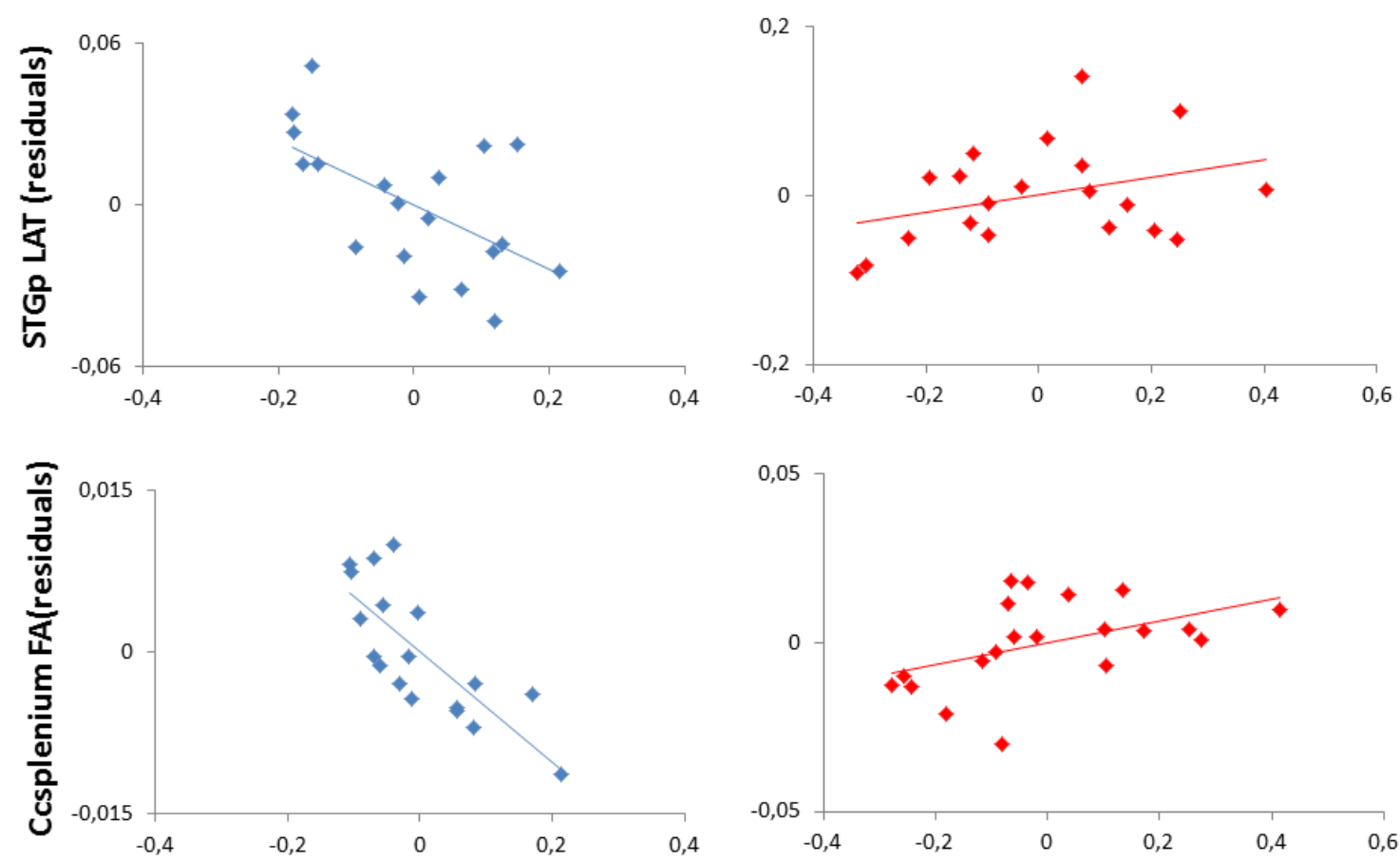

Figure 4 Scatterplots demonstrating the correlation between interhemispheric coherence to $20 \mathrm{~Hz} A M$ and FA-lateralization of STGp and FA of the CCsplenium in normal (NR) and dyslexic readers (DR). 


\section{Discussion}

In this study, we examined structural FA-lateralization of the dorsal white matter tract (i.e. the arcuate fasciculus) and its posterior endpoint (i.e. STGp) in a sample of normal reading and dyslexic adults for whom group differences in functional interhemispheric connectivity at $20 \mathrm{~Hz} A M$ have been reported previously (Poelmans et al., 2012). The unique contribution of the present study is that we investigated the association between white matter (using DTI) and interhemispheric coherence to $4 \mathrm{~Hz}$ and $20 \mathrm{~Hz}$ AM (using ASSR), representing modulation rates which are important for respectively syllable- and phoneme-level information in the speech envelope. Moreover, we examined whether this relation is different for dyslexic and normal readers. The combination of ASSR and DTI revealed that the degree of white matter lateralization in STGp and white matter integrity in CCsplenium was related to the amount of coherence between neural oscillations in both hemispheres. This association was only signficant for phonemic-rate envelope information (i.e. $20 \mathrm{~Hz}$ AM) and was opposite in normal reading versus dyslexic adults.

\subsection{Structural FA-lateralization in normal and dyslexic readers}

Previous studies have reported leftward volumetric asymmetries in the planum temporale and its surrounding areas (e.g. review Shapleske et al., 1999). Yet, limited evidence is available on lateralization of the microstructural properties of white matter, except for a post-mortem study showing more myelination in left versus right superior temporal cortex (Anderson et al., 1999) and DTI-studies showing higher FA in left versus right arcuate fasciculus (Thiebaut de Schotten et al., 2011; Catani et al., 2007; Büchel et al., 2004; Hagmann et al., 2006; Lebel \& Beaulieu, 2009; Nucifora et al., 2005; Powell et al., 2006). Our DTI-results confirm that normal readers demonstrate a leftwards FA-lateralization of the arcuate fasciculus, and extends these findings by also demonstrating a left FA-lateralization in STGp.

Second, our study shows that dyslexic readers do not display this left FA-asymmetry in STGp, and only to a smaller extent in the arcuate fasciculus. An abnormal structural lateralization in dyslexic readers is supported by early post-mortem and imaging studies that found a reduced volumetric asymmetry in the planum temporale, though more recent studies could not replicate this (Eckert et al., 2000). Concerning lateralization of microstructural white matter properties, only one DTI-study in dyslexic readers explicitly investigated FA-asymmetry in a dyslexic sample (Niogi \& McCandliss, 2006). In line with our results, they reported a decreased left FA-lateralization in the temporoparietal region for dyslexic readers, though this region was located medially to the STGp and the arcuate fasciculus where we identified lateralization differences. In sum, these group differences indicate that left FA-lateralization in posterior language areas might be important for an adequate reading 
performance. This questions whether there is also a link of FA-lateralization with the underlying processes of reading, such as auditory and phonological processing.

\subsection{Does FA-lateralization in STGp relate to functional phonemic- and syllabic-rate processing in normal and dyslexic readers?}

In the present study, the relation between FA-lateralization and functional phonemic- and syllabic-rate processing was investigated with ASSRs evoked by speech-weighted noise stimuli amplitude modulated at $4 \mathrm{~Hz}$ and $20 \mathrm{~Hz}$. The benefit of using speech-weighted noise that is amplitude modulated at specific frequencies is that (1) top-down effects of semantics are excluded since linguistic content is absent, and that (2) the neural responses to modulations that are specifically important for phoneme and syllable identification can be separated. Examining interhemispheric coherence in response to these auditory stimuli may thus provide unique information about processing low-level syllabic- and phonemic-rate envelope cues in speech.

Given the hierarchical model of auditory processing (Hickok et al., 2004), asymmetry of the STGp is expected to be more important for spectrotemporal acoustic analysis whereas asymmetry of the arcuate fasciculus is more important for high-level phonological processing. The latter was indeed supported by a recent DTI-study, which showed a link between good cognitive performance on a phonological test and leftward asymmetry of the arcuate fasciculus in a large group of typical developing children and adults (Lebel et al., 2009). The role of auditory processing in relation to FAlateralization, especially of the superior temporal cortex, was however still unexplored. Our study fills this gap by demonstrating that in normal readers, better phonemic-rate interhemispheric coherence related to leftwards FA-lateralization of the STGp. It has been hypothesized that left dominance in myelination underlies the left hemispheric preference for processing fast modulation (Zatorre \& Belin, 2001; Zatorre, Belin, \& Penhune, 2002) since the degree of myelination relates to nerve conduction velocity (Jack, Noble, \& Tsien, 1983). One previous study partly supports this hypothesis by demonstrating that more volume in the left auditory cortex, especially white matter volume, coheres with neural activity of temporal - and not of spectral - processing (Warrier et al., 2009). However, they used volumetric measures which are generally too gross to specifically examine the microstructural properties of white matter, such as myelination (Warrier et al., 2009). Given the positive relationship between FA and myelination (Hüppi et al., 1998; Werring, Clark, Barker, Thompson, \& Miller, 1999; Wimberger et al., 1995), the present study provides, for the first time, indirect evidence that more myelination (i.e. a higher FA) in the left relative to the right STGp is related to better neural processing of fast modulations (such as $20 \mathrm{~Hz}$ ). This finding supports multitime resolution models of speech processing (Hickok et al., 2007; Pöppel, 2003; Ghitza \& Greenberg, 2009), which predict that faster, phonemic-rate modulations are lateralized to the left hemisphere. 
We note however that in infants this functional left asymmetry for fast acoustic information is not consistently found, which leaves open the possibility that the left asymmetry we observed in adults is a reflection of particular learning mechanisms (for a review see Minagawa-Kawai, Cristià, \& Dupoux, 2011). This has important implications with regard to the interpretation of our results since left FAlateralization might be a consequence of reading experience rather than being at the basis of it. Future studies should therefore include preschoolers in order to determine whether a decreased leftwards FA-lateralization is an early marker of dyslexia or only present later in life as a consequence of reading failure.

Besides a leftwards dominance for fast modulation, the multi-time model predicts right hemispheric activation for slow, syllabic-rate modulations. This prediction was indeed confirmed in a large number of functional neuroimaging (Boemio et al., 2005; Belin et al., 1998; Telkemeyer et al., 2009), as well as EEG/MEG (Abrams et al., 2008; Britton, Blumstein, Myers, \& Grindrod, 2009; Luo \& Pöppel, 2007) studies, both in adults and in infants (Minagawa-Kawai et al., 2011). However, the relation between FA-lateralization measures and interhemispheric coherence to the syllabic rate did not reach significance level in our study. Although we do not have a final explanation for the lack of correlations in the present study, it may be that this functional right hemispheric lateralization for syllabic-rate modulations is not rooted in microstructural white matter properties such as myelination, or alternatively, in other brain regions than those we investigated. The latter is supported by studies demonstrating a different cortical locus for processing syllables and phonemes (Siok, Jin, Fletcher, \& Tan, 2003; Peeva et al., 2010). Another possible explanation is related to the age of the participants (i.e. adult participants). Given that adults' phonological awareness is more tuned to the phoneme level, it might be that - in parallel to their phonological development - adults only display correlations for phonemic-rate acoustic modulations, whereas infants or young children, who are more tuned to the syllable level, might also display correlations for the syllabic-rate modulations. The latter is indeed confirmed by previous studies in children who find correlations between neural processing of syllabic-rate amplitude changes and reading skills (e.g. Abrams, Nicol, Zecker, \& Kraus, 2009).

In dyslexic readers, no significant relation between FA-lateralization of STGp and interhemispheric coherence to $20 \mathrm{~Hz}$ or for $4 \mathrm{~Hz}$ AM was found. However, assuming the absolute correlation values, in dyslexic readers better phonemic-rate phase locking tends to relate to right lateralization of STGp. This reversed correlational pattern was confirmed by a significant interaction between group and interhemispheric coherence to $20 \mathrm{~Hz}$ AM for STGp. It is possible that poor readers implement a compensatory mechanism for a less effective left hemispheric network. If phonemic-rate acoustic information is insufficiently maintained to be integrated in the left dorsal pathway, it may be that dyslexic readers compensate this by depending more than normal readers 
on a right hemispheric network. Indeed, Hoeft et al. (2011) found that dyslexic readers use the right arcuate fasciculus to compensate their literacy problems. Compensation by activation in the right hemisphere is also commonly reported in a large variety of language deficits, including dyslexia. However, it rarely compensates all aspects of the behavioural profile (Kell et al., 2009; Lehongre et al., 2011; Preibisch et al., 2003). Second, it is possible that the formation of phonological representations of dyslexic readers relies on acoustic information of larger or smaller time chunks than the phonemic rate. Indeed, studies have shown that dyslexic readers phase locked better than normal readers to rates higher $(50 \mathrm{~Hz}-70 \mathrm{~Hz}$ : Lehongre et al., 2011) or lower (10 Hz: Hämäläinen et al., 2012) than the phonemic-rate. If phonological representations were based on acoustic information on a different timescale, they would be over- or underspecified and may be linked to the reading problems in dyslexia.

\subsection{Does FA in CCsplenium relate to functional phonemic- and syllabic- rate processing in normal and dyslexic readers?}

Given that dyslexic readers displayed a symmetric FA-pattern in STGp and no typical correlation with interhemispheric coherence to $20 \mathrm{~Hz}$ AM, we examined whether this was rooted in abnormalities of the callosal white matter fibers underlying this interhemispheric connection. A limitation of conventional DTI is that fibertracking is hindered by crossing fibers (Mori, 2007), which impedes the direct reconstruction of the fibers connecting the left and right STGp. Nevertheless, from post mortem research it is known that the fibers maintaining this connection run through the splenium of the CC (Westerhausen et al., 2009), and we therefore delineated the fibers passing through that region.

Our data showed a significant correlation between interhemispheric coherence to $20 \mathrm{~Hz} A M$ and FA in CCsplenium for both normal and dyslexic readers, which agrees with independent evidence that the integrity of the corpus callosum relates to functional interhemispheric coherence (Teipel et al., 2009; Pogarell et al., 2005). Given that fMRI (Shaywitz et al., 2003; 2007), DTI (Hoeft et al., 2011) and EEG (Dujardin et al., 2011) studies have suggested that dyslexic readers recruit right hemispheric areas to compensate for poor left hemisphere functioning, interhemispheric connectivity, both structurally and functionally, is especially important in dyslexia. Macrostructure studies of the CC in dyslexia are inconsistent, but several studies have shown a difference in the posterior $\mathrm{CC}$, particularly in the splenium (Duara et al., 1991; Rumsey et al., 1996). Also at the microstructural level, two recent DTI-studies have pointed out group differences in the posterior part of the $\mathrm{CC}$, with dyslexic readers having a higher FA than typical readers (Odegard, Farris, Ring, McColl, \& Black, 2009; Frey et al., 2008). Under the assumption that the CC plays an integrative role in hemispheric lateralization, it might be that a better structural connectivity of the splenium in dyslexic readers (as reflected by a 
higher FA) coheres with an atypical hemispheric asymmetry. Previous studies in a typical population have indeed shown an inverse relation between indices of lateralization and callosal size (Witelson \& Goldsmith, 1991) and callosal FA (Westerhausen et al., 2004; 2006; but see Putnam, Wig, Grafton, Kelley, \& Gazzaniga, 2008 for a positive relation). Given that the splenium region is crucial in connecting the left and right STGp (Westerhausen et al., 2009), it might thus be that the better structural connection of the splenium in dyslexic readers, as shown in previous DTI-studies, underlies the lower asymmetry we observed in STGp for our dyslexic readers. Although our results could not confirm FA group differences in CCsplenium, we did find an opposite correlational pattern between both groups. In dyslexic readers, FA in CCsplenium related positively with $20 \mathrm{~Hz}$ interhemispheric coherence, whereas this was negative in typical readers. Furthermore, we know from our ASSRresults that our dyslexic participants had lower interhemispheric coherence to $20 \mathrm{~Hz}$ AM than typical readers (Poelmans et al., 2012). Our present results indicate that those dyslexic readers that have stronger cohering neural oscillations in response to $20 \mathrm{~Hz}$ modulations also have a higher structural connectivity in the splenium of the CC as well as a more symmetric FA-pattern in STGp. Based on these results, we therefore hypothesize that, relative to normal readers, dyslexic readers compensate their phonemic-rate processing problems by relying more on the right STGp via a better structural connection in the splenium of the corpus callosum.

\subsection{An integrative model for dyslexia?}

By the combination of the present results together with those of previous studies in largely the same sample (Poelmans et al., 2012; Vandermosten et al., 2012) we suggest a speculative model on acoustic and phonological processing. First, the present study shows that neural interhemispheric coherence to phonemic-rate modulations depends in a typical population on a structural left dominance of white matter integrity in STGp (analogue to a functional leftward dominance for fast modulations) (Zatorre et al., 2008). Second, in a previous ASSR study, in which the present sample was extended up to 60 subjects, a link has been demonstrated between interhemispheric coherence to phonemic-rate modulations and speech-in-noise perception. Third, in a previous DTI-report involving the same sample as the present study (Vandermosten et al., 2012), it was demonstrated that speech-in-noise perception was positively correlated with the left and negatively with the right arcuate fasciculus, which suggests that optimal processing of the phonological aspects of speech perception depends on a structural left dominance of white matter integrity in the arcuate fasciculus.

Based on these three findings, we hypothesize that optimal left asymmetry of white matter integrity in STGp is crucial for an adequate neural transfer of phonemic-rate information, and that the quality by which these acoustic modulations are processed will reflect on further phonological processing. Anatomically, these phonological processes seem to take place in the arcuate fasciculus 
(which connects with STGP). This might imply that, analogue to the importance of left FAlateralization in STGp to optimally process phonemic-rate acoustic modulations, left FA-lateralization of the arcuate fasciculus might mediate processes depending on phonological representations, such as the perception of speech in noise. In contrast to this pattern, the present study shows that dyslexic readers are unable to develop sufficient FA-asymmetry in STGp, which corroborates their difficulties in neural transfer of phonemic-rate information. Given that left asymmetry in STGp is not significantly related to slow modulations, such as syllabic-rate information, the decreased left dominance in dyslexic readers seems not to affect auditory transfer of syllabic modulations and therefore no group differences are observed in neural interhemispheric coherence to syllabic-rate information. However, the decreased asymmetry in STGp and inadequate coherence to phonemicrate information can reflect on the further phonological analysis and on building well-specified phonological representations at the phoneme level. This might be evidenced by a decreased left asymmetry in the arcuate fasciculus and by their deviant performance on phonological tasks reported in Vandermosten et al. (2012). Thus, the consistent decreased left dominance demonstrated with the present results as well as a decreased functional (Poelmans et al., 2012) and structural (Vandermosten et al., 2012) intrahemispheric connectivity in the left hemisphere, might indicate that dyslexic readers tend to rely on a less developed network. Friederici (2011) has demonstrated that during typical development the functional language network evolves from reliance on interhemispheric towards left intrahemispheric connections. At the structural level, this finding is supported by developmental DTI-studies showing an early maturation of the CC and a late maturation of the arcuate fasciculus (Lebel et al., 2009). Though there is some evidence available in literature that the posterior part of the $\mathrm{CC}$ is more developed in dyslexic relative to typical readers, reflected by a higher FA (Odegard et al., 2009; Frey et al., 2008), the present study could not replicate this result. Nevertheless, our results do suggest a compensatory role of the CCsplenium in interhemispheric transfer of phonemic-rate modulation, possibly being more processed in the right STGp than in normal readers.

Although our study was able to demonstrate significant group differences (normal versus dyslexic readers) and relations between FA-lateralization and functional coherence to phonemic-rate modulations, we acknowledge that the obtained effects are only modest and not corrected for multiple comparisons. Given that we applied 6 General Linear Model tests to investigate DTI-group effects and its relation with ASSR-indices, a Bonferroni-correction would require a significance level of $p<0.008$. However, none of our obtained group effects (arcuate fasciculus: $p=0.043$; STGp: $p=0.021$ ) and their interaction with interhemispheric coherence to $20 \mathrm{~Hz}$ AM (STGp: $p=0.018$; CCsplenium: $p=0.022$ ) would survive this correction. Nevertheless, keeping in mind that the stringent Bonferonni-correction increases Type II errors (Perneger, 1998; Nakagawa, 2004), we 
believe that the obtained effects should not be interpreted as a nul-result, especially since the mirrored pattern of correlation in dyslexic versus normal readers is present in two regions. We do emphasize that results must be interpreted with caution and that more research is needed to investigate whether these effects can be replicated. Further development of the neurological techniques (e.g. ASSRs with high-density recordings and coherence analyses at the source level, or the possibility to reconstruct crossing fibers with DTI) might be necessary to reduce variability in the data and to further unravel the hypotheses we postulated here. In addition, no conclusions can be made on what is cause and consequence of reading experience since our study involved adult participants. The decreased left-lateralized myelination in dyslexic adults relative to normal reading adults could be an a priori difference that has caused the dyslexia, but it is equally likely to be a consequence of differences in reading experience and reading efficiency. Nevertheless, we believe that our results provide valuable and unique information on the interaction between structural white matter lateralization and processing of rapidly changing auditory information, which is of particular interest to theories of dyslexia.

\section{Conclusion}

The present study reveals that dyslexic readers have a decreased left lateralization of white matter integrity in auditory and dorsal language regions as well as a decreased neural coherence to phonemic but not for syllabic modulations. Given that previous studies indicate that neural processing of phonemic- versus syllabic-rate modulations is rooted in interhemispheric functional differences, the central aim of the present study was to investigate whether this lateralization pattern is also present at the structural level, specifically in white matter properties, and whether dyslexic readers display a deviant lateralization pattern. Our results reveal that normal readers display a link between neural interhemispheric response for phonemic-rate modulations and left FAlateralization in STGp, in line with functional lateralization studies, whereas dyslexic readers display the opposite pattern, with a tendency to relate more to right lateralization. Additionally, interhemispheric coherence to phonemic-rate modulations related differently to FA in CCsplenium for normal than for dyslexic readers. We conclude that deficient neural coherence to phonemic-rate modulations coheres with dyslexic's decreased left lateralization in STGp. This might in turn affect further phonological processing, which seems to be reflected in a decreased left lateralization of arcuate fasciculus. Possibly, dyslexic readers compensate their lack of left lateralization, necessary for phonemic-rate acoustic cues and phonological processing, by more interhemispheric white matter connections. 


\section{Acknowledgments}

The research was financed by the fund for Scientific Research Flanders, grants G0216.02, G0331.08 and G0920.12, and by a grant of the Research Council of Katholieke Universiteit Leuven, OT/07/034. Maaike Vandermosten is a junior research fellow of the Research Foundation Flanders. Hanne Poelmans is financed by a postdoctoral grant (PDMK/12/170). We are grateful to all participants in this study. Special thanks are due to Bart Boets and Heleen Luts for help and advice with data acquisition and processing and to Robert Luke for proofreading the manuscript. 


\section{References}

Abrams, D. A., Nicol, T., Zecker, S., \& Kraus, N. (2008). Right-hemisphere auditory cortex is dominant for coding syllable patterns in speech. Journal of Neuroscience, 28, 3958-3965.

Abrams, D. A., Nicol, T., Zecker, S., \& Kraus, N. (2009). Abnormal cortical processing of the syllable rate of speech in poor readers. Journal of Neuroscience, 29, 7686-7693.

Alexander, D. C., Pierpaoli, C., Basser, P. J., \& Gee, J. C. (2001). Spatial transformations of diffusion tensor magnetic resonance images. Medical Imaging, IEEE Transactions on, 20, 1131-1139.

Anderson, B., Southern, B. D., \& Powers, R. E. (1999). Anatomic asymmetries of the posterior superior temporal lobes: a postmortem study. Neuropsychiatry, neuropsychology, and behavioral neurology, 12, 247-254.

Anthony, J. L., Lonigan, C. J., Driscoll, K., Phillips, B. M., \& Burgess, S. R. (2003). Phonological sensitivity: A quasi-parallel progression of word structure units and cognitive operations. Reading Research Quarterly, 38, 470-487.

Barrick, T. R., Lawes, I. N., Mackay, C. E., \& Clark, C. A. (2007). White matter pathway asymmetry underlies functional lateralization. Cerebral Cortex, 17, 591-598.

Basser, P. J., Pajevic, S., Pierpaoli, C., Duda, J., \& Aldroubi, A. (2000). In vivo fiber tractography using DT-MRI data. Magnetic Resonance in Medicine, 44, 625-632.

Beaton, A. A. (1997). The relation of planum temporale asymmetry and morphology of the corpus callosum to handedness, gender, and dyslexia: a review of the evidence. Brain and Language, 60, 255-322.

Beaulieu, C. (2002). The basis of anisotropic water diffusion in the nervous system - a technical review. Nmr in Biomedicine, 15, 435-455.

Belin, P., McAdams, S., Smith, B., Savel, S., Thivard, L., Samson, S. et al. (1998). The functional anatomy of sound intensity discrimination. Journal of Neuroscience, 18, 6388-6394.

Benson, R. R., Richardson, M., Whalen, D. H., \& Lai, S. (2006). Phonetic processing areas revealed by sinewave speech and acoustically similar non-speech. Neuroimage, 31, 342-353.

Binder, J. R., Frost, J. A., Hammeke, T. A., Bellgowan, P. S. F., Springer, J. A., Kaufman, J. N. et al. (2000). Human temporal lobe activation by speech and nonspeech sounds. Cerebral Cortex, 10, 512528.

Boemio, A., Fromm, S., Braun, A., \& Pöppel, D. (2005). Hierarchical and asymmetric temporal sensitivity in human auditory cortices. Nature Neuroscience, 8, 389-395.

Boets, B., Vandermosten, M., Poelmans, H., Luts, H., Wouters, J., \& Ghesquière, P. (2011). Preschool impairments in auditory processing and speech perception uniquely predict future reading problems. Research in developmental disabilities, 32, 560-570.

Brauer, J., Anwander, A., \& Friederici, A. D. (2011). Neuroanatomical prerequisites for language functions in the maturing brain. Cerebral Cortex, 21, 459-466. 
Britton, B., Blumstein, S. E., Myers, E. B., \& Grindrod, C. (2009). The role of spectral and durational properties on hemispheric asymmetries in vowel perception. Neuropsychologia, 47, 1096-1106.

Büchel, C., Raedler, T., Sommer, M., Sach, M., Weiller, C., \& Koch, M. A. (2004). White matter asymmetry in the human brain: a diffusion tensor MRI study. Cerebral Cortex, 14, 945-951.

Catani, M., Allin, M. P. G., Husain, M., Pugliese, L., Mesulam, M. M., Murray, R. M. et al. (2007). Symmetries in human brain language pathways correlate with verbal recall. Proceedings of the National Academy of Sciences, 104, 17163-17168.

Chevillet, M. A., Jiang, X., Rauschecker, J. P., \& Riesenhuber, M. (2013). Automatic phoneme category selectivity in the dorsal auditory stream. The Journal of Neuroscience, 33, 5208-5215.

Corriveau, K. H., Goswami, U., \& Thomson, J. M. (2010). Auditory processing and early literacy skills in a preschool and kindergarten population. Journal of Learning Disabilities, 43, 369-382.

de Gelder, B. \& Vroomen, J. (1991). Phonological deficits: beneath the surface of reading-acquisition problems. Psychological Research, 53, 88-97.

Dehaene-Lambertz, G. \& Gliga, T. (2004). Common neural basis for phoneme processing in infants and adults. Journal of Cognitive Neuroscience, 16, 1375-1387.

Dehaene-Lambertz, G., Montavont, A., Jobert, A., Allirol, L., Dubois, J., Hertz-Pannier, L. et al. (2010). Language or music, mother or Mozart? Structural and environmental influences on infants' language networks. Brain and Language, 114, 53-65.

Dehaene-Lambertz, G., Pallier, C., Serniclaes, W., Sprenger-CharolleS, L., Jobert, A., \& Dehaene, S. (2005). Neural correlates of switching from auditory to speech perception. Neuroimage, 24, 21-33.

Depessemier, P. \& Andries, C. (2009). Gletschr, test voor gevorderd lezen en schrijven. Antwerp, Belgium: Garant.

Duara, R., Kushch, A., Grossglenn, K., Barker, W. W., Jallad, B., Pascal, S. et al. (1991). Neuroanatomical differences between dyslexic and normal readers on magnetic-resonance-imaging scans. Archives of Neurology, 48, 410-416.

Dujardin, T., Etienne, Y., Contentin, C., Bernard, C., Largy, P., Mellier, D. et al. (2011). Behavioral performances in participants with phonological dyslexia and different patterns on the N170 component. Brain and Cognition, 75, 91-100.

Eckert, M. A. \& Leonard, C. M. (2000). Structural imaging in dyslexia: The planum temporale. Mental Retardation and Developmental Disabilities Research Reviews, 6, 198-206.

Eden, G. F. \& Zeffiro, T. A. (1998). Neural systems affected in developmental dyslexia revealed by functional neuroimaging. Neuron, 21, 279-282.

Eluvathingal, T. J., Hasan, K. M., Kramer, L., Fletcher, J. M., \& Ewing-Cobbs, L. (2007). Quantitative diffusion tensor tractography of association and projection fibers in normally developing children and adolescents. Cerebral Cortex, 17, 2760-2768.

Farmer, M. E. \& Klein, R. M. (1995). The evidence for a temporal processing deficit linked to dyslexia: A Review. Psychonomic Bulletin \& Review, 2, 460-493. 
Frey, S., Campbell, J. S. W., Pike, G. B., \& Petrides, M. (2008). Dissociating the human language pathways with high angular resolution diffusion fiber tractography. The Journal of Neuroscience, 28, 11435-11444.

Friederici, A. D. (2011). The brain basis of language processing: from structure to function. Physiological Reviews, 91, 1357-1392.

Gaab, N., Gabrieli, J. D. E., Deutsch, G. K., Tallal, P., \& Temple, E. (2007). Neural correlates of rapid auditory processing are disrupted in children with developmental dyslexia and ameliorated with training: An fMRI study. Restorative Neurology and Neuroscience, 25, 295-310.

Ghitza, O. \& Greenberg, S. (2009). On the possible role of brain rhythms in speech perception: intelligibility of time-compressed speech with periodic and aperiodic insertions of silence. Phonetica, $66,113-126$

Goswami, U. (2002). In the beginning was the rhyme? A reflection on Hulme, Hatcher, Nation, Brown, Adams, and Stuart (2002). Journal of Experimental Child Psychology, 82, 47-57.

Goswami, U. \& East, M. (2000). Rhyme and analogy in beginning reading: Conceptual and methodological issues. Applied Psycholinguistics, 21, 63-93.

Goswami, U. (2011). A temporal sampling framework for developmental dyslexia. Trends in Cognitive Sciences, 15, 3-10.

Hagmann, P., Jonasson, L., Maeder, P., Thiran, J. P., Wedeen, V. J., \& Meuli, R. (2006). Understanding diffusion MR imaging techniques: from scalar diffusion-weighted imaging to diffusion tensor imaging and beyond. Radiographics, 26, S205-S223.

Hämäläinen, J. A., Rupp, A., Soltész, F., Szücs, D., \& Goswami, U. (2012). Reduced phase locking to slow amplitude modulation in adults with dyslexia: An MEG study. Neuroimage, 59, 2952-2961.

Herdman, A. T., Lins, O., Van Roon, P., Stapells, D. R., Scherg, M., \& Picton, T. W. (2002). Intracerebral sources of human auditory steady-state responses. Brain Topography, 15, 69-86.

Hickok, G. \& Pöppel, D. (2004). Dorsal and ventral streams: a framework for understanding aspects of the functional anatomy of language. Cognition, 92, 67-99.

Hickok, G. \& Pöppel, D. (2007). The cortical organization of speech processing. Nature Reviews Neuroscience, 8, 393-402.

Hoeft, F., McCandliss, B. D., Black, J. M., Gantman, A., Zakerani, N., Hulme, C. et al. (2011). Neural systems predicting long-term outcome in dyslexia. Proceedings of the National Academy of Sciences, 108, 361-366.

Hüppi, P. S., Maier, S. E., Peled, S., Zientara, G. P., Barnes, P. D., Jolesz, F. A. et al. (1998). Microstructural development of human newborn cerebral white matter assessed in vivo by diffusion tensor magnetic resonance imaging. Pedriatric Research, 44, 584-590.

Husain, F. T., Fromm, S. J., Pursley, R. H., Hosey, L. A., Braun, A. R., \& Horwitz, B. (2006). Neural bases of categorization of simple speech and nonspeech sounds. Human Brain Mapping, 27, 636-651.

Jack, J. J. B., Noble, D., \& Tsien, R. W. (1983). Electrical current flow in excitable cells. Oxford: Oxford University Press. 
Jäncke, L., Wüstenberg, T., Schulze, K., \& Heinze, H. J. (2002). Asymmetric hemodynamic responses of the human auditory cortex to monaural and binaural stimulation. Hearing Research, 170, 166-178.

Joanisse, M. F. \& Gati, J. S. (2003). Overlapping neural regions for processing rapid temporal cues in speech and nonspeech signals. Neuroimage, 19, 64-79.

John, M. S., Dimitrijevic, A., \& Picton, T. W. (2001). Weighted averaging of steady-state responses. Clinical Neurophysiology, 112, 555-562.

Kell, C. A., Neumann, K., von Kriegstein, K., Posenenske, C., von Gudenberg, A. W., Euler, H. et al. (2009). How the brain repairs stuttering. Brain, 132, 2747-2760.

Lambrecht Smith, S., Roberts, J. A., Locke, J. L., \& Tozer, R. (2010). An exploratory study of the development of early syllable structure in reading-impaired children. Journal of Learning Disabilities, 43, 294-307.

Landman, B. A., Farrell, J. A. D., Jones, C. K., Smith, S. A., Prince, J. L., \& Mori, S. (2007). Effects of diffusion weighting schemes on the reproducibility of DTI-derived fractional anisotropy, mean diffusivity, and principal eigenvector measurements at 1.5T. Neuroimage, 36, 1123-1138.

Lebel, C. \& Beaulieu, C. (2009). Lateralization of the arcuate fasciculus from childhood to adulthood and its relation to cognitive abilities in children. Human Brain Mapping, 30, 3563-3573.

Leemans, A. \& Jones, D. K. (2009). The B-matrix must be rotated when correcting for subject motion in DTI data. Magnetic Resonance in Medicine, 61, 1336-1349.

Lehongre, K., Ramus, F., Villiermet, N., Schwartz, D., \& Giraud, A. L. (2011). Altered low-gamma sampling in auditory cortex accounts for the three main facets of dyslexia. Neuron, 72, 1080-1090.

Leppänen, P. H. T., Hämäläinen, J. A., Salminen, H. K., Eklund, K. M., Guttorm, T. K., Lohvansuu, K. et al. (2010). Newborn brain event-related potentials revealing atypical processing of sound frequency and the subsequent association with later literacy skills in children with familial dyslexia. Cortex, 46, 1362-1376.

Lorenzi, C., Dumont, A., \& Fullgrabe, C. (2000). Use of temporal envelope cues by children with developmental dyslexia. Journal of Speech Language and Hearing Research, 43, 1367-1379.

Luo, H. \& Pöppel, D. (2007). Phase patterns of neuronal responses reliably discriminate speech in human auditory cortex. Neuron, 54, 1001-1010.

Maionchi-Pino, N., Magnan, A., \& Ecalle, J. (2010). The nature of the phonological processing in French dyslexic children: evidence for the phonological syllable and linguistic features' role in silent reading and speech discrimination. Annals of Dyslexia, 60, 123-150.

Makris, N., Kennedy, D. N., Mclnerney, S., Sorensen, A. G., Wang, R., Caviness, V. S. et al. (2005). Segmentation of subcomponents within the superior longitudinal fascicle in humans: a quantitative, in vivo, DT-MRI study. Cerebral Cortex, 15, 854-869.

Malmivuo, J. \& Plonsey, R. (1995). Bioelectromagnetism: Principles and applications of bioelectric and biomagnetic fields. New York, USA: Oxford University Press.

Matsumoto, R., Okada, T., Mikuni, N., Mitsueda-Ono, T., Taki, J., Sawamoto, N. et al. (2008). Hemispheric asymmetry of the arcuate fasciculus. Journal of Neurology, 255, 1703-1711. 
McAnally, K. I. \& Stein, J. F. (1997). Scalp potentials evoked by amplitude-modulated tones in dyslexia. Journal of Speech Language and Hearing Research, 40, 939-945.

Menell, P., McAnally, K. I., \& Stein, J. F. (1999). Psychophysical sensitivity and physiological response to amplitude modulation in adult dyslexic listeners. Journal of Speech Language and Hearing Research, 42, 797-803.

Meyer, M., Alter, K., Friederici, A. D., Lohmann, G., \& von Cramon, D. Y. (2002). FMRI reveals brain regions mediating slow prosodic modulations in spoken sentences. Human Brain Mapping, 17, 73-88.

Millman, R. E., Prendergast, G., Kitterick, P. T., Woods, W. P., \& Green, G. G. R. (2010). Spatiotemporal reconstruction of the auditory steady-state response to frequency modulation using magnetoencephalography. Neuroimage, 49, 745-758.

Minagawa-Kawai, Y., Cristià, A., \& Dupoux, E. (2011). Cerebral lateralization and early speech acquisition: a developmental scenario. Developmental cognitive neuroscience, 1, 217-232.

Morgan, A. E. \& Hynd, G. W. (1998). Dyslexia, neurolinguistic ability, and anatomical variation of the planum temporale. Neuropsychological reviews, 8, 79-93.

Mori, S. (2007). Introduction to diffusion tensor imaging. Amsterdam, The Netherlands: Elsevier.

Nakagawa, S. (2004). A farewell to Bonferroni: the problems of low statistical power and publication bias. Behavioral Ecology, 15, 1044-1045.

Niogi, S. N. \& McCandliss, B. D. (2006). Left lateralized white matter microstructure accounts for individual differences in reading ability and disability. Neuropsychologia, 44, 2178-2188.

Nittrouer, S. (2006). Children hear the forest (L). Journal of the Acoustical Society of America, 120, $1799-1802$.

Nucifora, P. G., Verma, R., Melhem, E. R., Gur, R. E., \& Gur, R. C. (2005). Leftward asymmetry in relative fiber density of the arcuate fasciculus. Neuroreport, 16, 791-794.

Nunez, P. L. \& Srinivasan, R. (2006). Electric fields of the brain: The neurophysics of EEG. (2nd ed.) New York, USA: Oxford University Press.

Obrig, H., Rossi, S., Telkemeyer, S., \& Wartenburger, I. (2010). From acoustic segmentation to language processing: evidence from optical imaging. Frontiers in Neuroenergetics, 23, 1-12.

Odegard, T. N., Farris, E. A., Ring, J., McColl, R., \& Black, J. (2009). Brain connectivity in non-reading impaired children and children diagnosed with developmental dyslexia. Neuropsychologia, 47, 19721977.

Okada, K., Rong, F., Venezia, J., Matchin, W., Hsieh, I. H., Saberi, K. et al. (2010). Hierarchical organization of human auditory cortex: evidence from acoustic invariance in the response to intelligible speech. Cerebral Cortex, 20, 2486-2495.

Oldfield, R. C. (1971). Assessment and analysis of handedness - Edinburgh inventory. Neuropsychologia, 9, 97-\&. 
Parker, G. J. M., Luzzi, S., Alexander, D. C., Wheeler-Kingshott, C. A. M., Ciccarelli, O., \& Lambon Ralph, M. A. (2005). Lateralization of ventral and dorsal auditory-language pathways in the human brain. Neuroimage, 24, 656-666.

Peelle, J. E., Eason, R. J., Schmitter, S., Schwarzbauer, C., \& Davis, M. H. (2010). Evaluating an acoustically quiet EPI sequence for use in fMRI studies of speech and auditory processing. Neuroimage, 52, 1410-1419.

Peeva, M. G., Guenther, F. H., Tourville, J. A., Nieto-Castanon, A., Anton, J. L., Nazarian, B. et al. (2010). Distinct representations of phonemes, syllables, and supra-syllabic sequences in the speech production network. Neuroimage, 50, 626-638.

Penhune, V. B., Zatorre, R. J., MacDonald, J. D., \& Evans, A. C. (1996). Interhemispheric anatomical differences in human primary auditory cortex: probabilistic mapping and volume measurement from magnetic resonance scans. Cerebral Cortex, 6, 661-672.

Perneger, T. (1998). What's wrong with Bonferroni adjustments. British Medical Journal, 316, 12361238.

Picton, T. W. (2011). Human auditory evoked potentials. San Diego, USA: Plural Publishing Inc.

Picton, T. W., John, M. S., Dimitrijevic, A., \& Purcell, D. (2003). Human auditory steady-state responses. International Journal of Audiology, 42, 177-219.

Poelmans, H., Luts, H., Vandermosten, M., Boets, B., Ghesquière, P., \& Wouters, J. (2012). Auditory steady state cortical responses indicate deviant phonemic-rate processing in adults with dyslexia. Ear and Hearing, 33, 134-143.

Pogarell, O., Teipel, S. J., Juckel, G., Gootjes, L., M+Âller, T., B++rger, K. et al. (2005). EEG coherence reflects regional corpus callosum area in Alzheimer's disease. Journal of Neurology, Neurosurgery \& Psychiatry, 76, 109-111.

Pöppel, D. (2003). The analysis of speech in different temporal integration windows: cerebral lateralization as 'asymmetric sampling in time'. Speech Communication, 41, 245-255.

Pöppel, D., Idsardi, W. J., \& van Wassenhove, V. (2008). Speech perception at the interface of neurobiology and linguistics. Philosophical Transactions of the Royal Society B-Biological Sciences, $363,1071-1086$.

Powell, H. W. R., Parker, G. J. M., Alexander, D. C., Symms, M. R., Boulby, P. A., Wheeler-Kingshott, C. A. M. et al. (2006). Hemispheric asymmetries in language-related pathways: A combined functional MRI and tractography study. Neuroimage, 32, 388-399.

Preibisch, C., Neumann, K., Raab, P., Euler, H. A., von Gudenberg, A. W., Lanfermann, H. et al. (2003). Evidence for compensation for stuttering by the right frontal operculum. Neuroimage, 20, 13561364.

Putnam, M. C., Wig, G. S., Grafton, S. T., Kelley, W. M., \& Gazzaniga, M. S. (2008). Structural organization of the corpus callosum predicts the extent and impact of cortical activity in the nondominant hemisphere. Journal of Neuroscience, 28, 2912-2928.

Ramus, F. \& Szenkovits, G. (2008). What phonological deficit? The Quarterly Journal of Experimental Psychology, 61, 129-141. 
Rauschecker, J. P. \& Scott, S. K. (2009). Maps and streams in the auditory cortex: nonhuman primates illuminate human speech processing. Nat Neurosci, 12, 718-724.

Richlan, F., Kronbichler, M., \& Wimmer, H. (2009). Functional abnormalities in the dyslexic brain: A quantitative meta-analysis of neuroimaging studies. Human Brain Mapping, 30, 3299-3308.

Rocheron, I., Lorenzi, C., Fullgrabe, C., \& Dumont, A. (2002). Temporal envelope perception in dyslexic children. Neuroreport, 13, 1683-1687.

Rodrigo, S., Naggara, O., Oppenheim, C., Golestani, N., Poupon, C., Cointepas, Y. et al. (2007). Human subinsular asymmetry studied by diffusion tensor imaging and fiber tracking. American Journal of Neuroradiology, 28, 1526-1531.

Rosen, S., Wise, R. J., Chadha, S., Conway, E. J., \& Scott, S. (2011). Hemispheric Asymmetries in Speech Perception: Sense, Nonsense and Modulations. PLoS ONE, 6, e24672.

Ruff, S., Cardebat, D., Marie, N., \& Démonet, J. F. (2002). Enhanced response of the left frontal cortex to slowed down speech in dyslexia: an fMRI study. Neuroreport, 13, 1285-1289.

Rumsey, J. M., Casanova, M., Mannheim, G. B., Patronas, N., DeVaughn, N., Hamburger, S. D. et al. (1996). Corpus callosum morphology, as measured with MRI, in dyslexic men. Biological Psychiatry, 39, 769-775.

Saur, D., Schelter, B., Schnell, S., Kratochvil, D., Küpper, H., Kellmeyer, P. et al. (2010). Combining functional and anatomical connectivity reveals brain networks for auditory language comprehension. Neuroimage, 49, 3187-3197.

Shannon, R. V., Zeng, F. G., Kamath, V., Wygonski, J., \& Ekelid, M. (1995). Speech recognition with primarily temporal cues. Science, 270, 303-304.

Shapleske, J., Rossell, S. L., Woodruff, P. W. R., \& David, A. S. (1999). The planum temporale: a systematic, quantitative review of its structural, functional and clinical significance. Brain Research Reviews, 29, 26-49.

Shaywitz, B. A. (1998). Functional organization of the brain of reading and dyslexia. Biological Psychiatry, 43, 210.

Shaywitz, B. A., Shaywitz, S. E., Pugh, K. R., Mencl, W. E., Fulbright, R. K., Skudlarski, P. et al. (2002). Disruption of posterior brain systems for reading in children with developmental dyslexia. Biological Psychiatry, 52, 101-110.

Shaywitz, B. A., Skudlarski, P., Holahan, J. M., Marchione, K. E., Constable, R. T., Fulbright, R. K. et al. (2007). Age-related changes in reading systems of dyslexic children. Annals of Neurology, 61, 363370.

Shaywitz, S. E., Shaywitz, B. A., Fulbright, R. K., Skudlarski, P., Mencl, W. E., Constable, R. T. et al. (2003). Neural systems for compensation and persistence: young adult outcome of childhood reading disability. Biological Psychiatry, 54, 25-33.

Siok, W. T., Jin, Z., Fletcher, P., \& Tan, L. H. (2003). Distinct brain regions associated with syllable and phoneme. Human Brain Mapping, 18, 201-207.

Snowling, M. (2000). Dyslexia. (2nd ed.) Oxford, UK: Blackwell. 
Stuart, G. W., McAnally, K. I., Mckay, A., Johnston, M., \& Castles, A. (2006). A test of the magnocellular deficit theory of dyslexia in an adult sample. Cognitive Neuropsychology, 23, 12151229.

Swan, D. \& Goswami, U. (1997). Phonological awareness deficits in developmental dyslexia and the phonological representations hypothesis. Journal of Experimental Child Psychology, 66, 18-41.

Tallal, P. (1980). Auditory temporal perception, phonics, and reading disabilities in children. Brain and Language, 9, 182-198.

Teipel, S. J., Pogarell, O., Meindl, T., Dietrich, O., Sydykova, D., Hunklinger, U. et al. (2009). Regional networks underlying interhemispheric connectivity: An EEG and DTI study in healthy ageing and amnestic mild cognitive impairment. Human Brain Mapping, 30, 2098-2119.

Telkemeyer, S., Rossi, S., Koch, S. P., Nierhaus, T., Steinbrink, J., Pöppel, D. et al. (2009). Sensitivity of newborn auditory cortex to the temporal structure of sounds. Journal of Neuroscience, 29, 1472614733.

Tervaniemi, M. \& Hugdahl, K. (2003). Lateralization of auditory-cortex functions. Brain Research Reviews, 43, 231-246.

Thiebaut de Schotten, M., ffytche, D. H., Bizzi, A., Dell'Acqua, F., Allin, M., Walshe, M. et al. (2011). Atlasing location, asymmetry and inter-subject variability of white matter tracts in the human brain with MR diffusion tractography. Neuroimage, 54, 49-59.

Van Dun, B., Verstraeten, S., Alaerts, J., Luts, H., Moonen, M., \& Wouters, J. (2008). A flexible research platform for multi-channel auditory steady-state response measurements. Journal of Neuroscience Methods, 169, 239-248.

van Wieringen, A. \& Wouters, J. (2008). LIST and LINT: Sentences and numbers for quantifying speech understanding in severely impaired listeners for Flanders and the Netherlands. International Journal of Audiology, 47, 348-355.

Vandermosten, M., Boets, B., Poelmans, H., Sunaert, S., Wouters, J., \& Ghesquière, P. (2012). A tractography study in dyslexia: neuroanatomic correlates of orthographic, phonological and speech processing. Brain, 135, 935-948.

Vandermosten, M., Boets, B., Sunaert, S., Wouters, J., \& Ghesquière, P. (2012). A qualitative and quantitative review of diffusion tensor imaging studies in reading and dyslexia. Neuroscience \& Biobehavioral Reviews, 36, 1532-1552.

Vellutino, F. R., Fletcher, J. M., Snowling, M. J., \& Scanlon, D. M. (2004). Specific reading disability (dyslexia): what have we learned in the past four decades? Journal of Child Psychology and Psychiatry, 45, 2-40.

Vernooij, M. W., Smits, M., Wielopolski, P. A., Houston, G. C., Krestin, G. P., \& van der Lugt, A. (2007). Fiber density asymmetry of the arcuate fasciculus in relation to functional hemispheric language lateralization in both right- and left-handed healthy subjects: A combined fMRI and DTI study. Neuroimage, 35, 1064-1076.

Wakana, S., Caprihan, A., Panzenboeck, M. M., Fallon, J. H., Perry, M., Gollub, R. L. et al. (2007). Reproducibility of quantitative tractography methods applied to cerebral white matter. Neuroimage, 36, 630-644. 
Warrier, C., Wong, P., Penhune, V., Zatorre, R., Parrish, T., Abrams, D. et al. (2009). Relating structure to function: Heschl's gyrus and acoustic processing. Journal of Neuroscience, 29, 61-69.

Wechsler, D. (1992). Wechsler Adult Intelligence Scale (3rd Edition NL). Londen, UK: The Psychological Corporation.

Weiss, S. \& Mueller, H. M. (2003). The contribution of EEG coherence to the investigation of language. Brain and Language, 85, 325-343.

Werring, D. J., Clark, C. A., Barker, G. J., Thompson, A. J., \& Miller, D. H. (1999). Diffusion tensor imaging of lesions and normal-appearing white matter in multiple sclerosis. Neurology, 52, 16261632.

Westerhausen, R., Grüner, R., Specht, K., \& Hugdahl, K. (2009). Functional relevance of interindividual differences in temporal lobe callosal pathways: A DTI tractography study. Cerebral Cortex, 19, 1322-1329.

Westerhausen, R., Kreuder, F., Santos Sequeira, S. D., Walter, C., Woerner, W., Wittling, R. A. et al. (2006). The association of macro- and microstructure of the corpus callosum and language lateralisation. Brain and Language, 97, 80-90.

Westerhausen, R., Kreuder, F., Sequeira, S. D. S., Walter, C., Woerner, W., Wittling, R. A. et al. (2004). Effects of handedness and gender on macro- and microstructure of the corpus callosum and its subregions: a combined high-resolution and diffusion-tensor MRI study. Cognitive Brain Research, 21, 418-426.

Wimberger, D. M., Roberts, T. P., Barkovich, A. J., Prayer, L. M., Moseley, M. E., \& Kucharczyk, J. (1995). Identification of "premyelination" by diffusion-weighted MRI. Journal of computer assisted tomography, 19, 28-33.

Witelson, S. F. (1989). Hand and sex differences in the isthmus and genu of the human corpus callosum. Brain, 112, 799-835.

Witelson, S. F. \& Goldsmith, C. H. (1991). The relationship of hand preference to anatomy of the corpus callosum in men. Brain Research, 545, 175-182.

Witton, C., Stein, J. F., Stoodley, C. J., Rosner, B. S., \& Talcott, J. B. (2002). Separate influences of acoustic AM and FM sensitivity on the phonological decoding skills of impaired and normal readers. Journal of Cognitive Neuroscience, 14, 866-874.

Witton, C., Talcott, J. B., Hansen, P. C., Richardson, A. J., Griffiths, T. D., Rees, A. et al. (1998). Sensitivity to dynamic auditory and visual stimuli predicts nonword reading ability in both dyslexic and normal readers. Current Biology, 8, 791-797.

Wong, F. C. K., Chandrasekaran, B., Garibaldi, K., \& Wong, P. C. M. (2011). White matter anisotropy in the ventral language pathway predicts sound-to-word learning success. The Journal of Neuroscience, $31,8780-8785$.

Zaehle, T., Geiser, E., Alter, K., Jancke, L., \& Meyer, M. (2008). Segmental processing in the human auditory dorsal stream. Brain Research, 1220, 179-190. 
Zaehle, T., Wustenberg, T., Meyer, M., \& Jancke, L. (2004). Evidence for rapid auditory perception as the foundation of speech processing: a sparse temporal sampling fMRI study. European Journal of Neuroscience, 20, 2447-2456.

Zatorre, R. J. \& Belin, P. (2001). Spectral and temporal processing in human auditory cortex. Cerebral Cortex, 11, 946-953.

Zatorre, R. J., Evans, A. C., Meyer, E., \& Gjedde, A. (1992). Lateralization of phonetic and pitch discrimination in speech processing. Science, 256, 846-849.

Zatorre, R. J. \& Gandour, J. T. (2008). Neural specializations for speech and pitch: moving beyond the dichotomies. Philosophical Transactions of the Royal Society B-Biological Sciences, 363, 1087-1104.

Zatorre, R. J. (1988). Pitch perception of complex tones and human temporal-lobe function. The Journal of the Acoustical Society of America, 84, 566-572.

Zatorre, R. J., Belin, P., \& Penhune, V. B. (2002). Structure and function of auditory cortex: music and speech. Trends in Cognitive Sciences, 6, 37-46.

Zatorre, R. J., Meyer, E., Gjedde, A., \& Evans, A. C. (1996). PET studies of phonetic processing of speech: review, replication, and reanalysis. Cerebral Cortex, 6, 21-30.

Ziegler, J. C. \& Goswami, U. (2005). Reading acquisition, developmental dyslexia, and skilled reading across languages: A psycholinguistic grain size theory. Psychological Bulletin, 131, 3-29. 